\title{
Trajectory Reconstruction and Uncertainty Analysis using Mars Science Laboratory Pre-Flight Scale Model Aeroballistic Testing
}

\author{
Rafael A. Lugo ${ }^{*}$, Robert H. Tolson ${ }^{\dagger}$ \\ North Carolina State University, Raleigh, NC 27695 \\ and \\ Mark Schoenenberger ${ }^{\ddagger}$ \\ NASA Langley Research Center, Hampton, VA 23681
}

\begin{abstract}
As part of the Mars Science Laboratory (MSL) trajectory reconstruction effort at NASA Langley Research Center, free-flight aeroballistic experiments of instrumented MSL scale models was conducted at Aberdeen Proving Ground in Maryland. The models carried an inertial measurement unit (IMU) and a flush air data system (FADS) similar to the MSL Entry Atmospheric Data System (MEADS) that provided data types similar to those from the MSL entry. Multiple sources of redundant data were available, including tracking radar and on-board magnetometers. These experimental data enabled the testing and validation of the various tools and methodologies that will be used for MSL trajectory reconstruction. The aerodynamic parameters Mach number, angle of attack, and sideslip angle were estimated using minimum variance with $a$ priori to combine the pressure data and pre-flight computational fluid dynamics (CFD) data. Both linear and non-linear pressure model terms were also estimated for each pressure transducer as a measure of the errors introduced by CFD and transducer calibration. Parameter uncertainties were estimated using a "consider parameters" approach.
\end{abstract}

\section{Introduction}

Mars Science Laboratory (MSL) was a NASA Flagship mission that arrived at Mars in August 2012. The MSL entry vehicle, the largest and most advanced vehicle ever sent to Mars, carried a variety of entry-phase sensors in the form of the MSL Entry, Descent and Landing Instrumentation (MEDLI) suite. ${ }^{1}$ As a subset of MEDLI, the MSL heat shield had seven flush atmospheric data ports connected to pressure transducers for measuring pressure during the EDL sequence. Together, these pressure transducers formed the Mars Entry Atmospheric Data System (MEADS). ${ }^{2}$

Trajectory reconstructions of Earth-based vehicles using flush air data systems (FADS) similar in function to MEADS have been successfully conducted. ${ }^{3,4,5}$ Free-flight aeroballistic experiments utilizing scale models of the Crew Exploration Vehicle (CEV) were previously conducted at Aberdeen Proving Ground (APG) in Maryland from 2006 to 2009, as a joint effort between NASA Langley Research Center (LaRC) and the US Army Research Laboratory (ARL). ${ }^{6}$ The success of these experiments has led to further experimentation using scale models of the MSL entry vehicle, which is the subject of the present paper.

\section{Experiment Design}

Aeroballistic experimental firings of the MSL scale models were conducted by the ARL Flight Sciences Branch at the APG Transonic Experimental Facility (TEF) in June 2012. Computer-Aided Design/Manufacturing (CAD/CAM), model fabrication, and telemetry instrumentation was performed by the ARL Guidance Technology Branch. The models were encased in sabots and fired from a gun with a 7-inch barrel diameter, at initial velocities of

\footnotetext{
* Graduate Research Assistant, Department of Mechanical and Aerospace Engineering, AIAA Student Member

${ }^{\dagger}$ Research Professor, Department of Mechanical and Aerospace Engineering, AIAA Associate Fellow

$\$$ Aerospace Engineer, Atmospheric Flight and Entry Systems Branch, AIAA Member
}

1

American Institute of Aeronautics and Astronautics 
up to Mach 4.0. The gun shown in Figure 1 had been part of the High Altitude Research Project (HARP), a program started in the 1960s to characterize atmosphere conditions at high altitudes. ${ }^{7}$

On-site assets included tracking radar, fixed-head radar, and high-speed digital tracking (or flight follower) cameras operated by Aberdeen Test Center (ATC) personnel. The radar systems were used to obtain a "truth" trajectory in the form of inertial position and velocity. Cameras were used to visually verify vehicle behavior near the muzzle exit.

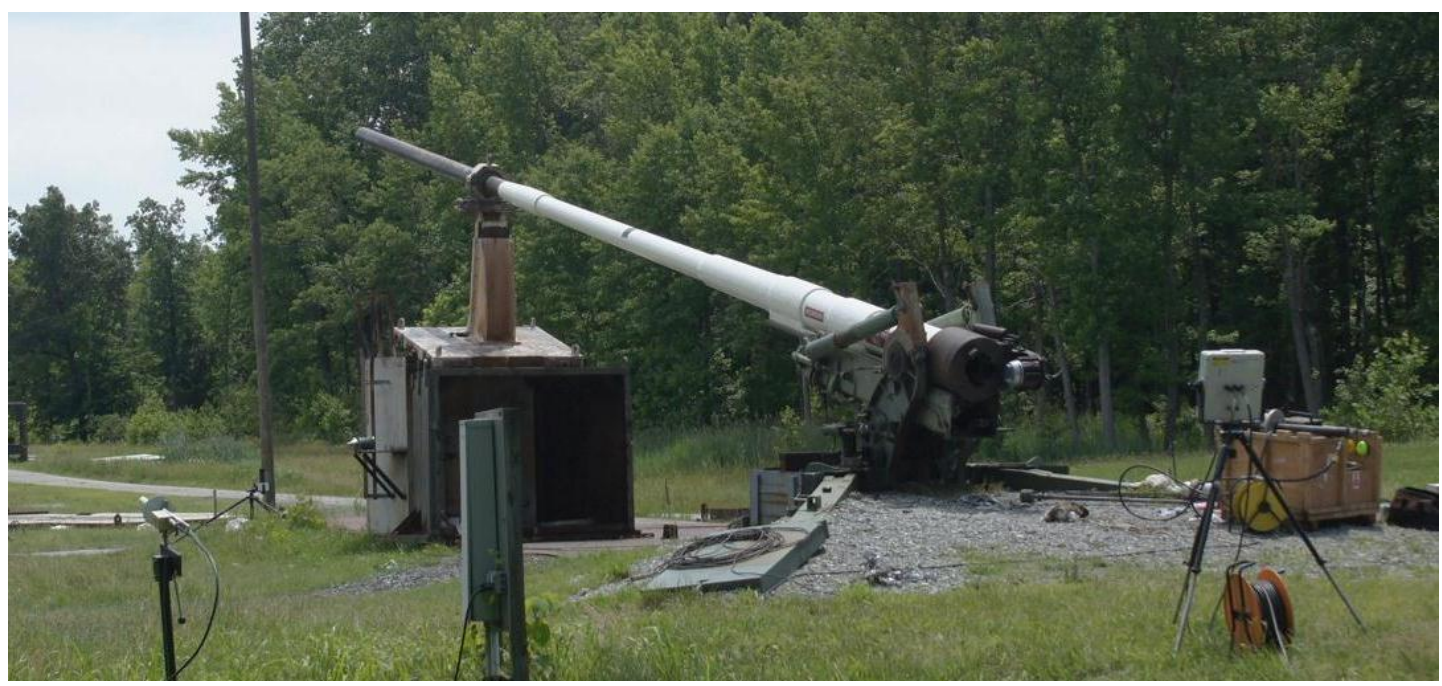

Figure 1. Aeroballistic experiment setup at Aberdeen Proving Ground, MD.

Table 1 lists relevant information regarding the aeroballistic experiments for the three MSL-PTM flights. Note that one model was axisymmetric and the remaining two were lifting bodies (i.e. they were manufactured with offset centers of gravity).

Table 1. Experiment information for MSL-PTM flights.

\begin{tabular}{l|ccc}
\hline \hline & MSL5PTM6 & MSL8PTM9 & MSL7PTM5 \\
\hline \hline Name & MSL-PTM-01, Flight 01 & MSL-PTM-02, Flight 02 & MSL-PTM-03, Flight 03 \\
Date fired & 13 June 2012 & 18 June 2012 & 18 June 2012 \\
Configuration & Axisymmetric & Lifting & Lifting \\
In-flight mass (kg) & 7.257 & 7.348 & 7.348 \\
Initial Mach number & $\sim 3.76$ & $\sim 3.81$ & $\sim 3.66$ \\
\hline \hline
\end{tabular}

\section{A. Pressure Transducer Module}

The MSL pressure transducer modules (MSL-PTM, Figure 2) were scale models of MSL constructed primarily of stainless steel. Tungsten ballasts were embedded in the vehicles to reproduce center of mass offsets, shown in the CAD drawing in Figure 3. Results from a model manufactured with no center of mass offset (MSL-PTM-01) are the primary subject of the present paper.

All models were fitted with ARL's multifunctional instrumentation and data acquisition system (MIDAS), shown at right in Figure 2 with an IMU containing two three-axis accelerometers, two three-axis rate gyros, and two three-axis magnetometers. ${ }^{8}$ Seven pressure ports and transducers were arranged on the heat shield in the MEADS configuration, shown in Figure 4. Because there were a total of ten available instrumentation channels, three additional pressure ports $(8,9$ and 10) were placed on the heat shield to provide further redundant data and potentially improve solutions. 


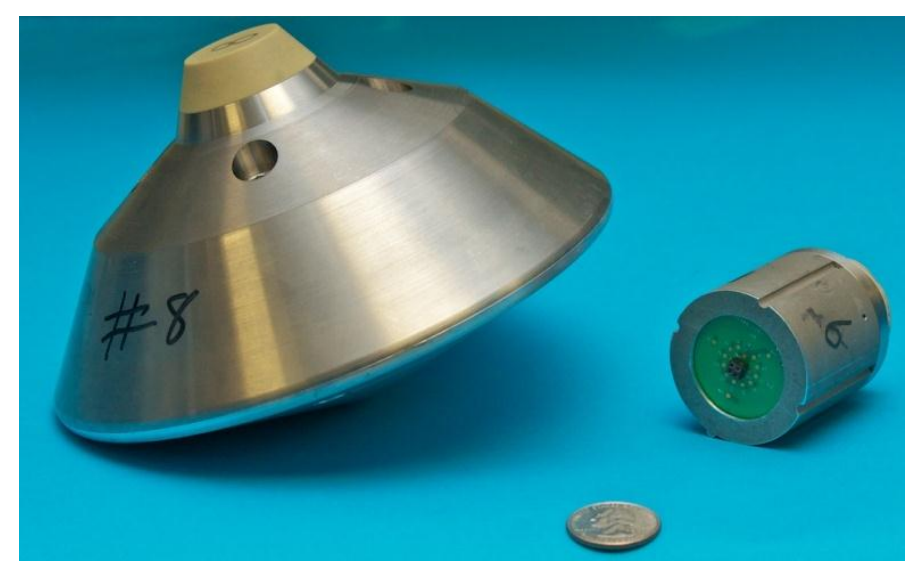

Figure 2. Mars Science Laboratory Pressure Transducer Module (MSL-PTM, left) and sensor/telemetry package (right). United States quarter shown for scale.

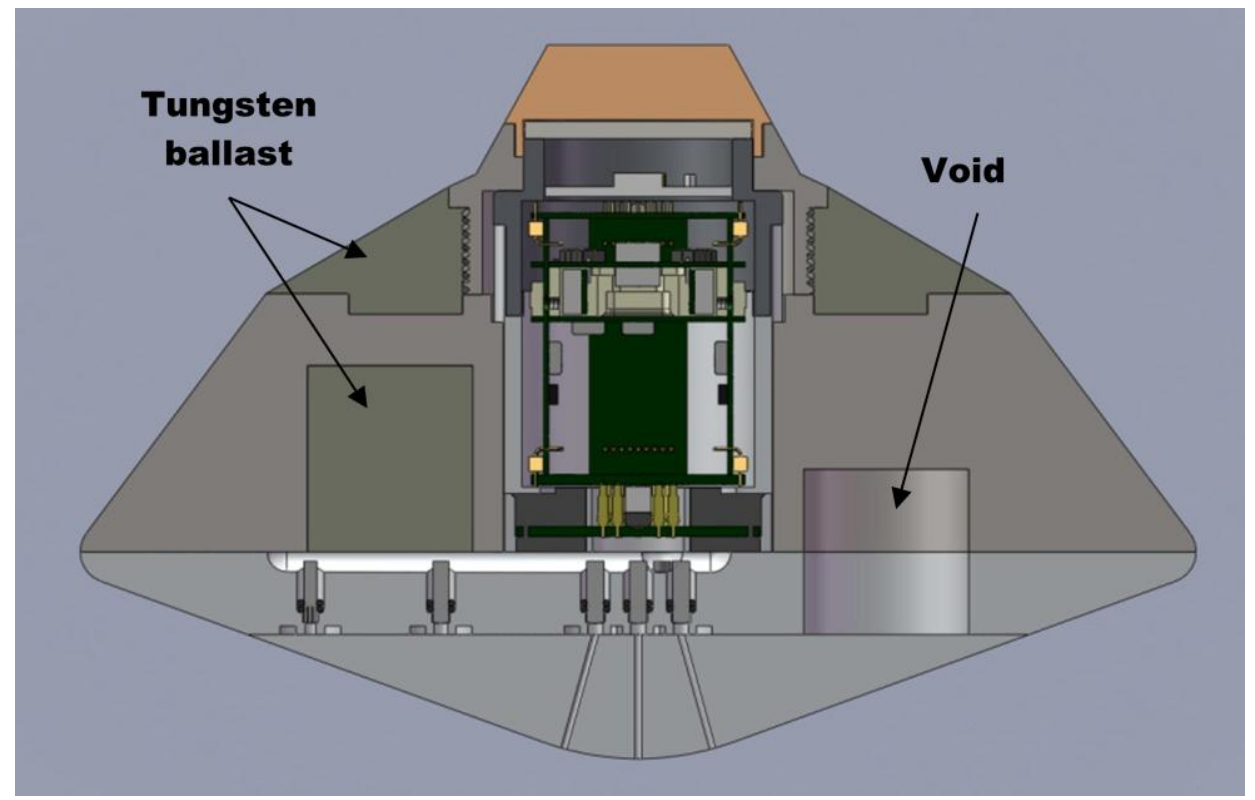

Figure 3. MSL-PTM cross-section.

Analysis of previous aeroballistic experiments ${ }^{9}$ showed that the accelerometers and rate gyroscopes in the CEVPTM IMU were of insufficient quality to reconstruct a valid inertial navigation trajectory. Specifically, a combination of biases and scale factors on the accelerations and rates that satisfied the expected trajectory could not be found, due primarily to the high sensitivity of the solutions to various error sources. However, attitude rates from the IMU were validated using rates derived from the magnetometers, and Mach number was validated using radar data. Based on the results of the CEV-PTM experiments, the MSL-PTM IMU sensor compliment was changed to carry redundant accelerometers and rate gyros. Furthermore, the MSL flight IMUs are of significantly higher quality than the IMUs aboard the PTM. 


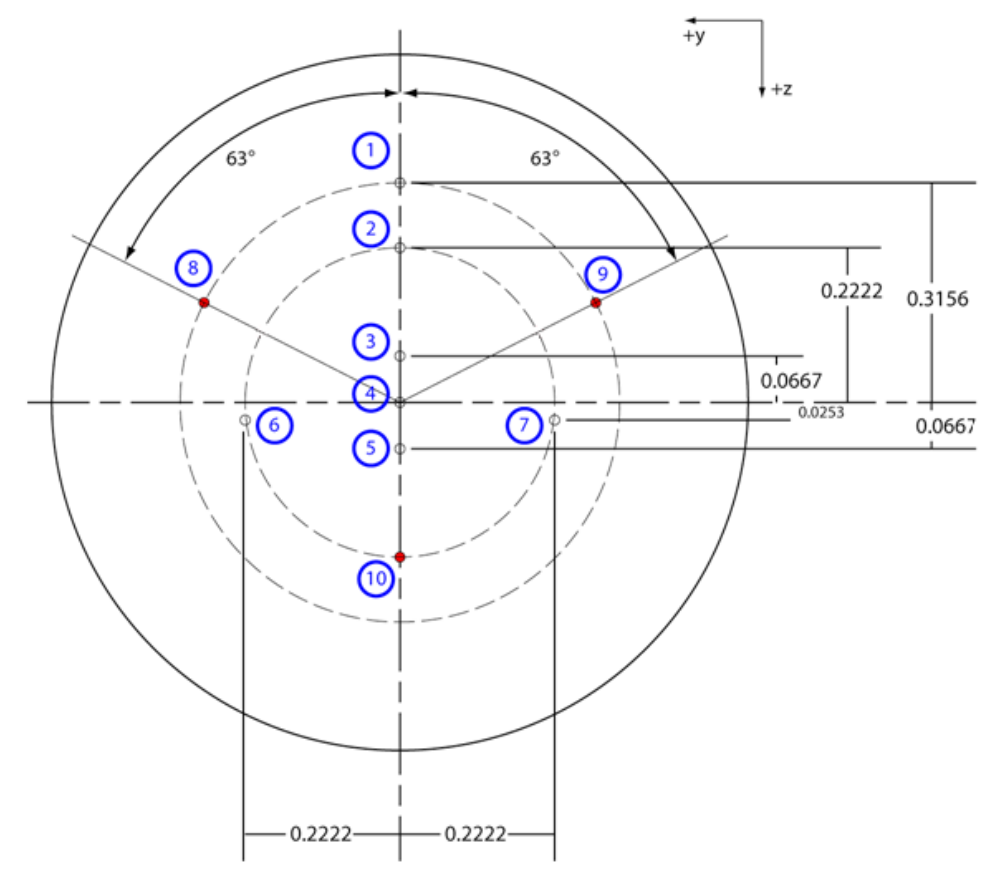

Figure 4. Dimensionless MEADS port locations on MSL heat shield. Locations normalized by model diameter.

\section{B. Pressure Calibration}

The pressure transducers were calibrated at LaRC in April 2012. Each PTM was placed inside a vessel that was brought to one of nine designated calibration pressure points, at which point the pressure (observed as 12-bit words) was recorded for ten seconds. The recording was then stopped, the vessel was brought to a different calibration pressure point, and the process repeated. A Design of Experiments (DOE) approach was used in which the pressure calibration points were set randomly.

A linear pressure model was used for pre-flight calibration:

$$
P_{\text {mod }}=k c_{0}+k c_{1} \tilde{P}
$$

where $c_{0}$ is the bias in bits, $c_{1}$ is the scale factor, $k$ is conversion from bits to psi, $\tilde{P}$ is the pressure observed by the transducer in bits, and $P_{\text {mod }}$ is the model pressure in psi. Calibration scale factors were all uniformly close to 0.15 , and biases ranged from 110 to $130 \mathrm{psi}$. This significant bias was due to the transducers returning non-zero values at zero pressure.

Maximum calibration pressure was $150 \mathrm{psi}$, due to limitations of the calibration vessel hardware. Though this was significantly lower than the $\sim 250$ psi (1.75 million Pa) that would later observed by some of the transducers, non-linear behavior was not expected as the transducers are rated to 500 psi by the manufacturer. Analysis comparisons between the model and CFD-based pressure on CEV flights, however, showed non-linear behavior in the pressure residuals, indicating that the pressure transducers were in fact exhibiting non-linear behavior in the high pressure regimes.9 Furthermore, the pressure transducers could not be re-calibrated post-flight since the PTM was not recovered. For these reasons both linear and non-linear pressure model terms were estimated post-flight.

\section{Radar Calibration}

The radar equipment at APG was calibrated prior to experiment execution, in March and April 2012. A $120 \mathrm{~mm}$ artillery gun was used to fire small scale model MSL slugs, saving the use of instrumented models. An example of these scale models, and their sabots, is shown in Figure 5.

Prior to firing, the radar attitude locked in an orientation that pointed its main lobe (the region of strongest possible signal return) to an area through which the vehicle was expected to fly. Ideally, the vehicle traveled through this area, the radar unlocked, and began tracking. The goal of the radar calibration was to determine the time delay, from muzzle exit, to unlock the tracking radar, as well as an elevation that was low enough to capture the slug close 
to muzzle exit, but high enough to prevent the radar from locking onto one of the falling sabot petals (seen prelaunch at left in Figure 5 and post-launch at right in Figure 12).

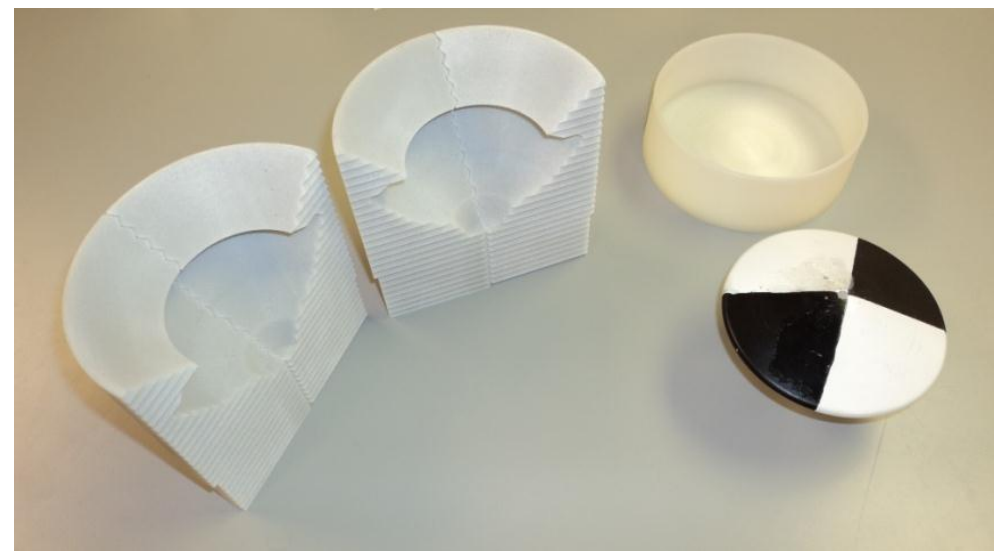

Figure $5.120 \mathrm{~mm}$ radar calibration sabot and blank calibration slug. Slug is at right with heat shield facing up.

Radar calibration began with tracking rapid-prototyped lifting cylindrical slugs $120 \mathrm{~mm}$ in diameter designed to roughly mimic the trajectory of the MSL-PTM. Once these were successfully tracked, lifting MSL slugs were used. After several shots and radar adjustments, the radar was able to successfully track the MSL slugs, and the radar settings from these calibrations were used on subsequent 7-inch instrumented flights.

\section{Trajectory Reconstruction}

\section{A. Coordinate Frames}

Three coordinate frames were used in this analysis: the inertial frame, body frame, and wind frame. A flat, nonrotating Earth was assumed. In the present paper, when vector frames must be explicitly identified, the exponent $n$ will denote the inertial frame and $b$ will denote the body frame.

The inertial frame (also referred to as the range coordinate frame or Earth-fixed frame) has the origin at the muzzle exit. The $\mathrm{X}$ axis is aligned with the direction of the gun. The $\mathrm{XZ}$ plane is tangent to the flat Earth and the $\mathrm{XY}$ plane is perpendicular to the flat Earth (i.e., aligned with the direction of gravity).

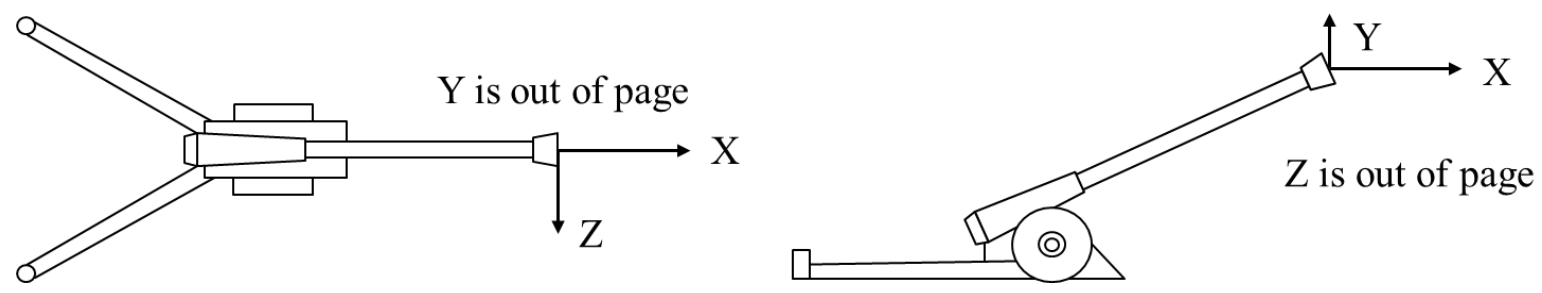

Figure 6. Inertial (range) coordinate system.

The body frame was chosen to coincide with the MSL aerodynamic frame, as shown in Figure 7, which has the origin at the center of gravity. 


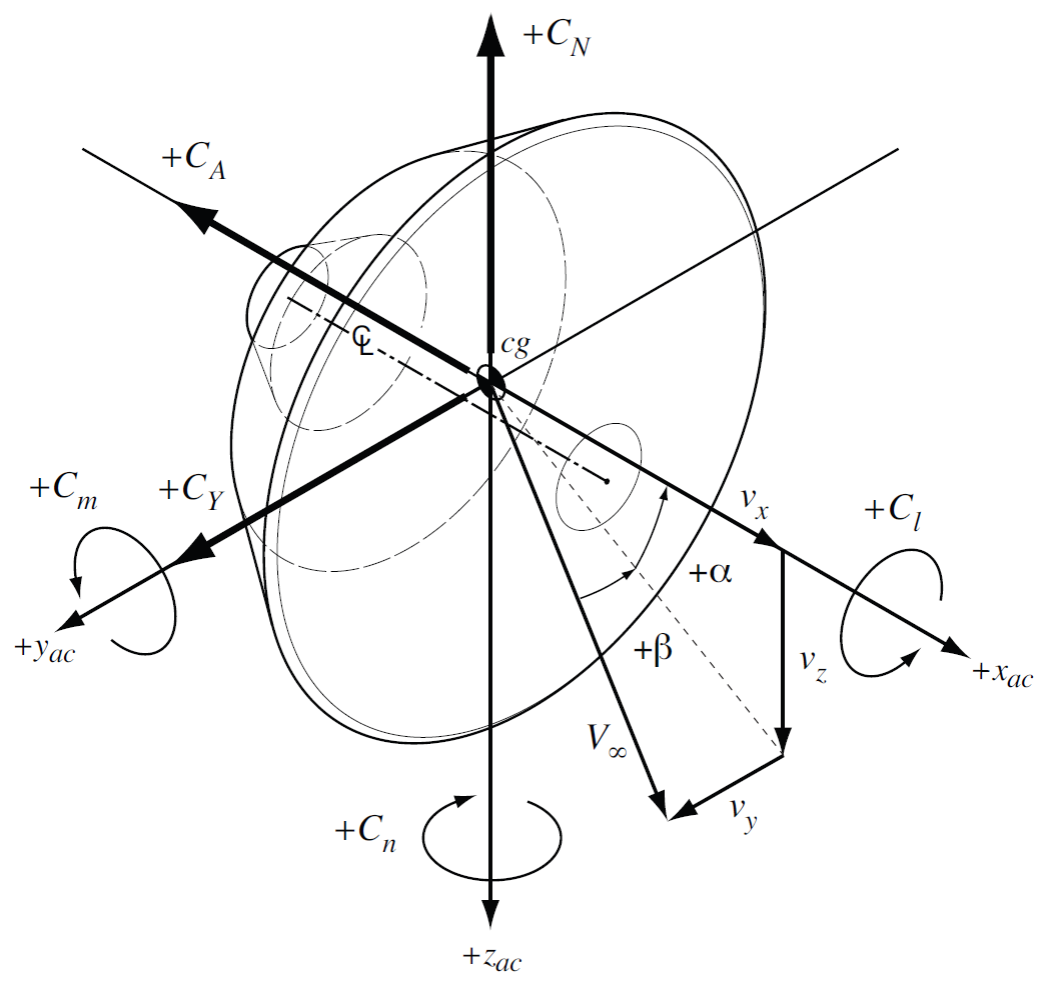

Figure 7. Mars Science Laboratory aerodynamic $\left(x_{a c}, y_{a c}, z_{a c}\right)$ coordinate system with aerodynamic force coefficients $\left(C_{A}, C_{N}, C_{Y}\right)$, angle of attack $\alpha$, sideslip angle $\beta$, and wind-relative velocity vector $V_{\infty}$.

Reconstruction of the MSL-PTM trajectory was performed using an inertial navigation approach, integrating the observed accelerations and angular rates in the appropriate reference frame. Observations were made in the IMU reference frame, and were transformed to the body frame using a transformation matrix:

$$
\mathbf{a}^{b}=\mathrm{C}^{b i} \mathbf{a}^{i}
$$

where $\mathbf{a}^{i}$ is the observation vector as sensed by the IMU, $\mathrm{C}^{b i}$ is the direction cosine matrix that transforms from the IMU frame $i$ to the body frame $b$, and $\mathbf{a}^{b}$ is the observation vector in the body frame.

The estimated aerodynamic parameters consisted of Mach number $M$, angle of attack $\alpha$, and sideslip angle $\beta$. The latter two are often referred to as wind angles. The wind angles describe the difference between the body and wind axes, which both have origins at the vehicle center of gravity. The wind $x$-axis points along the velocity vector $\mathbf{V}=\left[\begin{array}{lll}u & v & w\end{array}\right]^{\mathrm{T}}\left(V_{\infty}\right.$ in Figure 7$)$. The wind angles can be written in terms of the components of the body frame velocity vector:

$$
\begin{array}{ll}
\tan \alpha=\frac{w}{u} & -\pi \leq \alpha \leq \pi \\
\sin \beta=\frac{v}{V} & -\frac{\pi}{2} \leq \beta \leq \frac{\pi}{2}
\end{array}
$$

where $V=|\mathbf{V}|=\sqrt{u^{2}+v^{2}+w^{2}}$ and $u>0$. The velocities in the body frame, likewise, can be written in terms of the flight angles:

$$
\begin{aligned}
& u=V \cos \beta \cos \alpha \\
& v=V \sin \beta \\
& w=V \cos \beta \sin \alpha
\end{aligned}
$$

Mach number is derived from the total velocity:

$$
M=V / c
$$

where $c$ is the local speed of sound. 


\section{B. Inertial Navigation Solution}

Inertial navigation is a deterministic trajectory reconstruction method that uses data from the inertial measurement unit (also called the inertial navigation system or the inertial reference unit) to determine the vehicle position and velocity in an inertial frame. ${ }^{10}$ For this type of reconstruction, the required data types are body frame accelerations $\mathbf{a}=\left[\begin{array}{lll}a_{x} & a_{y} & a_{z}\end{array}\right]^{\mathrm{T}}$ and angular rotation rates $\boldsymbol{\omega}=\left[\begin{array}{lll}\omega_{x} & \omega_{y} & \omega_{z}\end{array}\right]^{\mathrm{T}}$.

The inertial navigation process requires that center of mass body accelerations be integrated in the state equations, rather than the sensed accelerations. A transformation was therefore made to account for accelerations due to angular motion of the vehicle by first computing the vector from the center of mass to the IMU:

$$
\Delta \mathbf{r}_{c}=\mathbf{r}_{\mathrm{CM}}^{b}-\mathbf{r}_{\mathrm{IMU}}^{b}
$$

The computed center of mass body accelerations are then

$$
\mathbf{a}_{\mathrm{CM}}=\tilde{\mathbf{a}}-\left[\tilde{\boldsymbol{\omega}} \times \Delta \mathbf{r}_{c}+\tilde{\boldsymbol{\omega}} \times\left(\tilde{\boldsymbol{\omega}} \times \Delta \mathbf{r}_{c}\right)\right]
$$

where $\tilde{\mathbf{a}}$ and $\tilde{\boldsymbol{\omega}}$ are the sensed body acceleration and angular rate vectors, respectively.

The rate of change of the quaternion was integrated, given an initial attitude, to obtain the quaternion history using the differential equation

$$
\left[\begin{array}{l}
\dot{q}_{0} \\
\dot{q}_{1} \\
\dot{q}_{2} \\
\dot{q}_{3}
\end{array}\right]=\frac{1}{2}\left[\begin{array}{cccc}
0 & -\omega_{1} & -\omega_{2} & -\omega_{3} \\
\omega_{1} & 0 & \omega_{3} & -\omega_{2} \\
\omega_{2} & -\omega_{3} & 0 & \omega_{1} \\
\omega_{3} & \omega_{2} & -\omega_{1} & 0
\end{array}\right]\left[\begin{array}{l}
q_{0} \\
q_{1} \\
q_{2} \\
q_{3}
\end{array}\right]
$$

where the quaternion nomenclature is

$$
q=q_{0}+q_{1} \hat{i}+q_{2} \hat{j}+q_{3} \hat{k}
$$

and $q_{0}$ denotes the scalar component of the quaternion and the magnitude (scalar norm) of $q$ is 1 . The quaternion history was converted to a direction cosine matrix history $\mathrm{C}^{n b}$, which was in turn used to transform the accelerations a from the body frame $b$ to the inertial frame $n$ :

$$
\mathbf{a}^{n}=\mathrm{C}^{n b} \mathbf{a}_{\mathrm{CM}}^{b}
$$

The inertial acceleration was integrated once to get the inertial velocity, and integrated again to get the inertial position. The state dynamics were modeled using the following equations of motion in the inertial frame:

$$
\begin{aligned}
& \dot{x}=u \\
& \dot{y}=v \\
& \dot{z}=w \\
& \dot{u}=a_{x}^{n}+g_{x}^{n} \\
& \dot{v}=a_{y}^{n}+g_{y}^{n} \\
& \dot{w}=a_{z}^{n}+g_{z}^{n}
\end{aligned}
$$

where $u, v$, and $w$ are the velocity vector components, $a_{x}, a_{y}$, and $a_{z}$ are the acceleration vector components, and $g_{x}, g_{y}$, and $g_{z}$ are the gravity vector components. Since the projectile in this case traveled less than two kilometers, a simple gravity model was used, $\mathbf{g}^{n}=\left[\begin{array}{lll}0 & -9.81 & 0\end{array}\right]^{\mathrm{T}} \mathrm{m} / \mathrm{s}$.

The reconstructed trajectory from the inertial navigation solution is shown in Figure 8. There are clear deviations of the inertial navigation solution from the radar trajectory. Initial condition errors are prevalent since the initial attitude of the vehicle is poorly known. By the time the vehicle has exited the muzzle and sensor noise has decreased from the firing explosion to the point of reading usable data, the vehicle has changed its orientation by an unknown amount. High-speed video camera footage (see Figure 12) can help attitude reconstruction, but in practice even minor errors in initial conditions prevent an accurate reconstruction. ${ }^{9}$ 

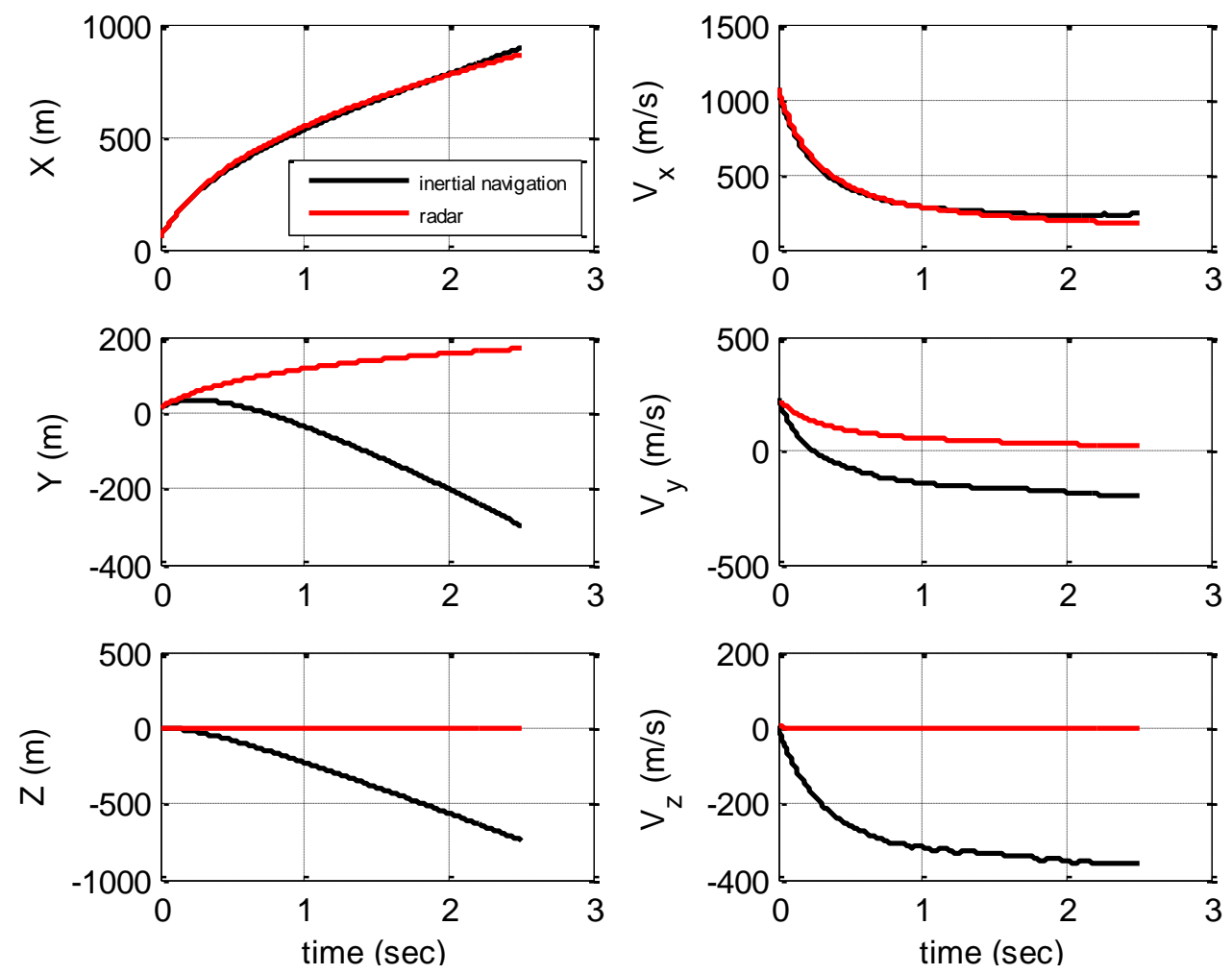

Figure 8. Inertial navigation solution compared to radar trajectory, MSL-PTM-01.

\section{Parameter Estimation}

In general, entry vehicle aerodynamic parameters can be recovered from FADS pressure data. Methods using weighted least squares have been demonstrated, ${ }^{4,11}$ but in the present paper the focus will be on minimum variance with a priori, a rigorous, statistical extension of the least squares estimator that does not assume diagonal weighting. ${ }^{12}$ With non-linear problems, it is advantageous to use a differential correction approach that incrementally updates the solution by using prior estimates $\mu$ to efficiently compute the new estimate. The minimum variance estimator, when combined with a priori and differential correction, is

$$
\Delta \hat{\mathbf{x}}_{k}=\left(\mathrm{A}^{\mathrm{T}} \Gamma_{\varepsilon}^{-1} \mathrm{~A}+\Gamma_{\mu}^{-1}\right)^{-1}\left(\mathrm{~A}^{\mathrm{T}} \Gamma_{\varepsilon}^{-1} \Delta \mathbf{y}+\Gamma_{\mu}^{-1}\left(\boldsymbol{\mu}-\hat{\mathbf{x}}_{k}\right)\right)
$$

where A is the sensitivity matrix, $\Gamma_{\mu}$ is the covariance matrix associated with the a priori state estimates $\mu, \Delta \mathbf{y}$ is the vector of the differences between observed and computed data, and $\hat{\mathbf{x}}_{k+1}=\hat{\mathbf{x}}_{k}+\Delta \hat{\mathbf{x}}_{k}$ provides the state estimation update. Note the limiting cases: (1) if the a priori knowledge is poor, then $\Gamma_{\mu}^{-1} \rightarrow 0$ and the solution reduces to the weighted least squares estimate, and (2) if the measurement knowledge is poor, then $\Gamma_{\varepsilon}^{-1} \rightarrow 0$ and the solution reduces to $\hat{\mathbf{x}}_{k}=\boldsymbol{\mu}$. The covariance of the final estimate is

$$
\Gamma_{\mathrm{x}}=\mathrm{V}(\hat{\mathbf{x}}-\mathbf{x})=\left(\mathrm{A}^{\mathrm{T}} \Gamma_{\varepsilon}^{-1} \mathrm{~A}+\Gamma_{\mu}^{-1}\right)^{-1}
$$

Minimum variance with a priori and differential correction has been demonstrated as an effective method for obtaining both state estimates and meaningful statistics from FADS. ${ }^{9}$

\section{A. Flush Air Data System Solution}

The FADS-based solution was obtained by converging on a set of aerodynamic parameters Mach number, angle of attack, and angle of sideslip that minimized the residuals between observed and CFD model pressures in a sequential manner. This can be thought of as attempting to fit a point in $M-\alpha-\beta$ space to the ten observed pressures at any given time. The aerodynamic parameters, being functions of pressure, changed at every time step and were defined as local parameters. Pressure transducer biases, scale factors, and quadratic and cubic terms applied to the entire dataset and were defined as global parameters. 
There were two loops in the estimation process: within the outer loop was the inner loop where local parameters were estimated at each data point. After this inner loop processed every data point across time, the outer loop batchestimated the global parameters. This process, illustrated in a flowchart in Figure 9, was repeated until convergence. Interpolated pressure values from the pre-flight CFD aerodynamic database were compared to the observed FADS pressure values shown in Figure 10.

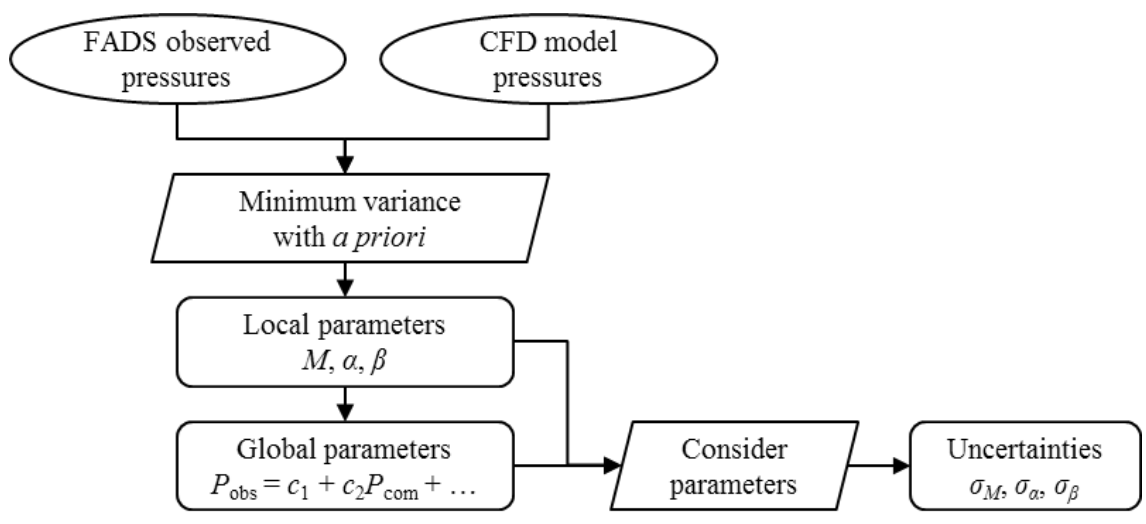

Figure 9. FADS- and CFD-based estimation process.
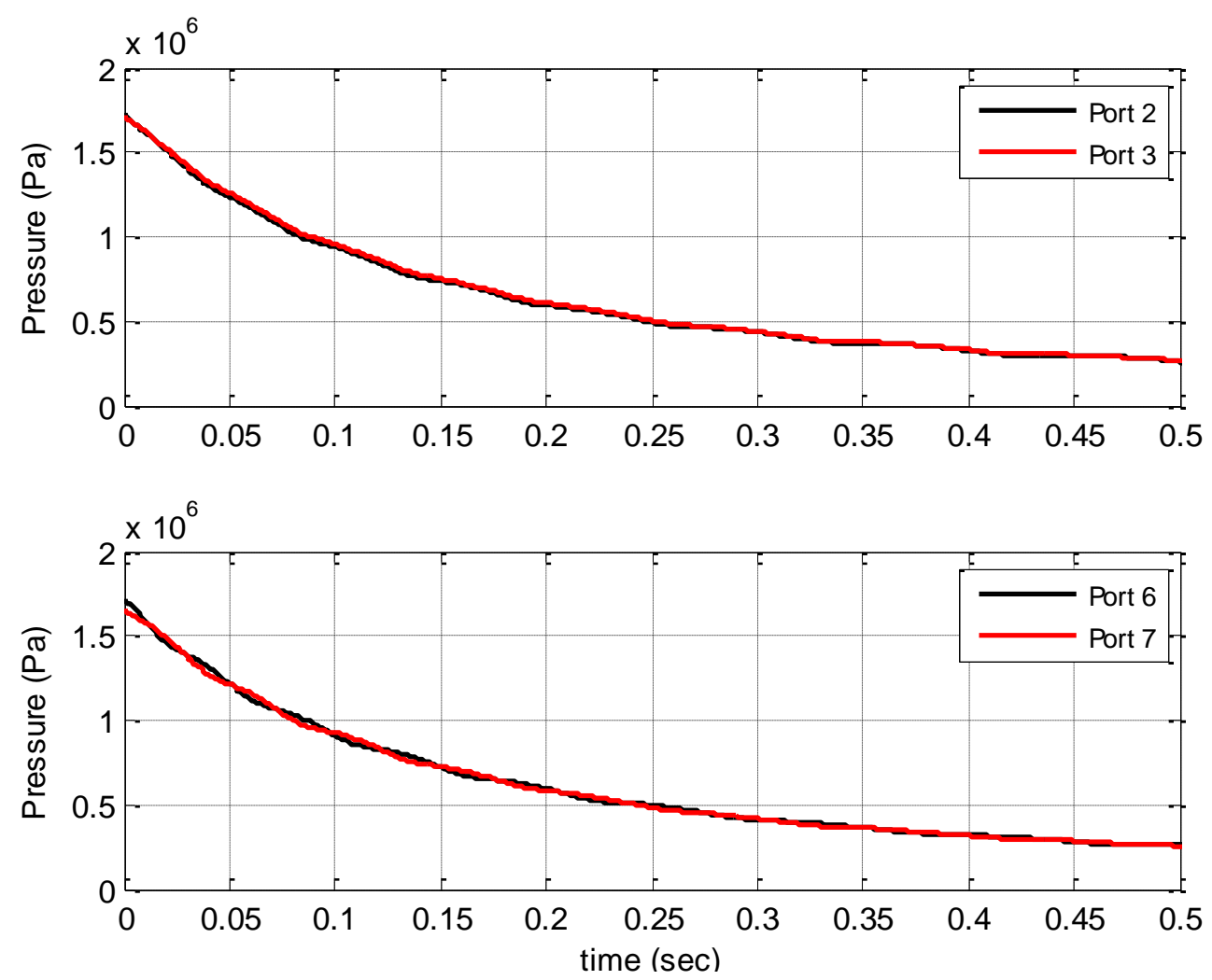

Figure 10. Observed FADS pressures, MSL-PTM-01.

Biases, scale factors, and higher-order terms for each pressure transducer were batch estimated after convergence of the local parameters using this method. ${ }^{9}$ The pressure model is

$$
P_{\text {model }}=c_{0}+c_{1} P_{\mathrm{CFD}}+c_{2} P_{\mathrm{CFD}}^{2}+c_{3} P_{\mathrm{CFD}}^{3}
$$


where $P_{\mathrm{CFD}}$ is the pressure recovered from the CFD database, $c_{0}$ is the bias, $c_{1}$ is the scale factor, $c_{2}$ is the quadratic term, and $c_{3}$ is the cubic term. The a priori uncertainty for each pressure transducer was $3000 \mathrm{~Pa}$ and was assumed to be constant throughout the flight.

A priori estimates and uncertainties used in the estimation process are listed in Table 2 . The uncertainties were chosen to be small enough so that the minimum variance algorithm did not diverge, but large enough so that a local minimum difference between observed and CFD pressures could be found in the $M-\alpha-\beta$ space.

Table 2. A priori estimates and uncertainties for MSL-PTM-01.

\begin{tabular}{l|cc}
\hline \hline Parameter & Initial estimate & Uncertainty \\
\hline \hline Mach number & 3.76 & 0.01 \\
Angle of attack & $0.0^{\circ}$ & $2.0^{\circ}$ \\
Angle of sideslip & $0.0^{\circ}$ & $1.0^{\circ}$ \\
\hline \hline
\end{tabular}

Figure 11 shows the estimated flight parameters for MSL-PTM flight 01. The first solution (black line) was estimated without data from ports 1 and 5. These two channels were lost during launch and telemetered unusable data. Note that these ports are along the vertical axis (see Figure 4) and contributed primarily to the estimate of angle of attack.

The second solution (red line) excluded data from ports 1 and 5 and ports 8-10. Recall that ports 1-7 were the MEADS configuration, and ports 8-10 were added specifically for these aeroballistic experiments. It can be seen that this angle of attack solution is biased compared to the first solution, which includes one more vertical port (port 10). There is more confidence in the solution with the additional pressure ports; having ports 1 and 5 would have improved the result even further. Higher uncertainty estimates were obtained when other vertical ports were excluded, which will be discussed in the next section.
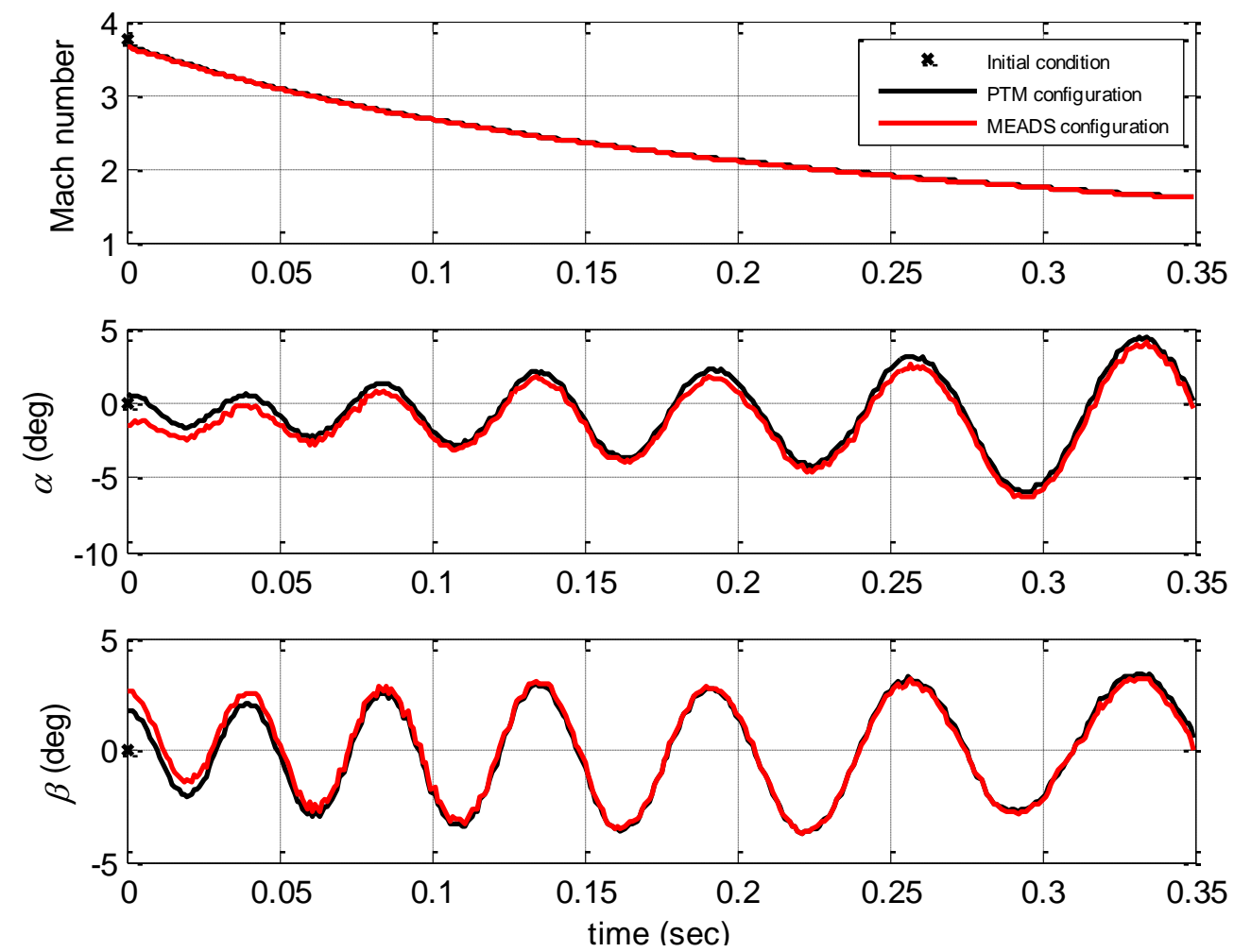

Figure 11. FADS-derived aerodynamic parameters, MSL-PTM-01. Both solutions exclude ports 1 and 5.

The behavior of the wind angles in Figure 11 generally matched theory in that an axisymmetric rigid body with zero trim will tend to oscillate about zero angle of attack and sideslip angle. This behavior was also supported by the high-speed video taken near the muzzle exit, which showed the vehicle exiting smoothly with little to no visible 
oscillation. A frame of this video is shown in Figure 12. (The CEV models, by way of comparison, were designed with CG offsets to fly at a predicted trim angle of approximately $18^{\circ}$, and therefore oscillated about that angle. ${ }^{9}$ )

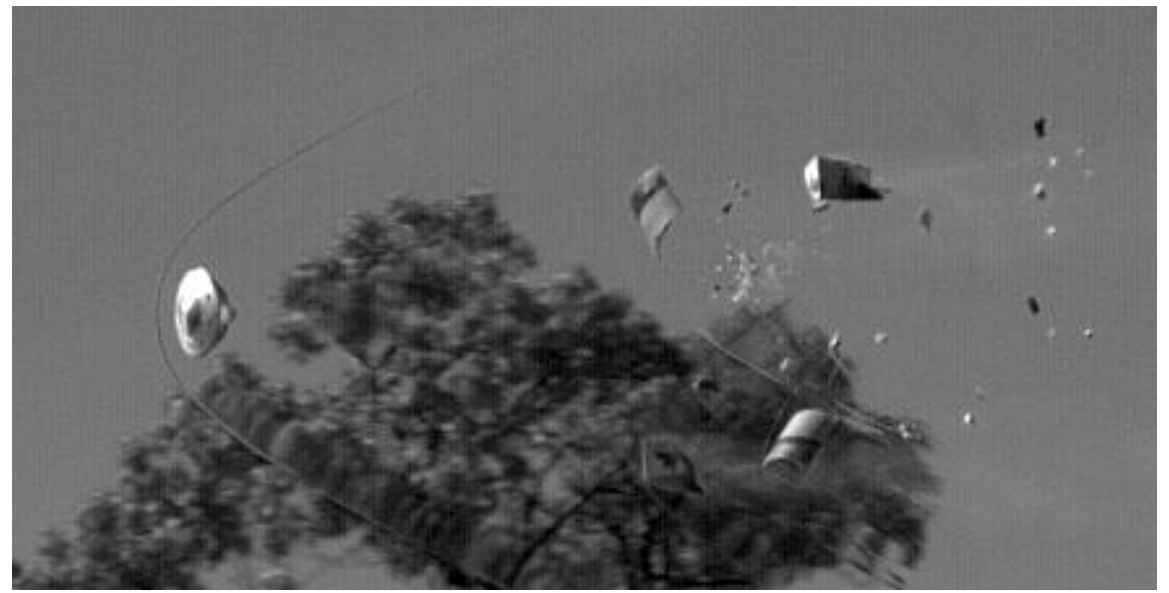

Figure 12. MSL-PTM-01 (left) just after muzzle exit. Sabot fragments at right. Frame taken from high-speed flight follower video.

Table 3 lists estimated biases, scale factors, and higher order terms for the PTM port configuration (all ports except 1 and 5). Note that the maximum observed pressure was approximately 1.75 million $\mathrm{Pa}$ (compare to the full scale pressure of 3.45 million $\mathrm{Pa}$, or $500 \mathrm{psi}$ ).

The transducer bias estimates listed in the table are at or below the transducer $1 \sigma$ uncertainty of $3000 \mathrm{~Pa}$, and the scale factors are uniformly below $1 \%$, indicating that the pre-flight transducer calibrations were accurate. The quadratic terms produce a $\Delta P$ of up to $35000 \mathrm{~Pa}$ on port 6 , which is about $2.0 \%$ of the maximum observed pressure. The cubic terms, similarly, produce a $\Delta P$ of up to $55000 \mathrm{~Pa}$ on Port 2, which is about $3.2 \%$ of the maximum observed pressure. The quadratic and cubic terms nominally produce $\Delta P$ s that are below $2 \%$ of the maximum observed pressure, which are not insignificant. Recall that the pressure transducers were calibrated only up to $60 \%$ of the maximum observed pressure due to calibration hardware limitations, and that potential changes in transducer behavior could not be measured after firing. Thus, any residual calibration errors are absorbed by the higher-order terms.

Table 3. Pressure transducer model coefficients, cubic model, MSL-PTM-01, PTM port configuration. The last two columns are the contributions of the quadratic and cubic terms as a percentage of maximum observed pressure of $\mathbf{1 . 7 5}$ million Pa.

\begin{tabular}{c|cccc}
\hline \hline Port & Bias (Pa) & S.F. & Quadratic & Cubic \\
\hline \hline 2 & 1685 & 1.0039 & $1.69 \%$ & $3.17 \%$ \\
\hline 3 & -3037 & 0.9910 & $1.41 \%$ & $2.90 \%$ \\
\hline 4 & -2816 & 0.9937 & $0.50 \%$ & $1.09 \%$ \\
\hline 6 & -1599 & 0.9987 & $2.03 \%$ & $1.32 \%$ \\
\hline 7 & 858 & 0.9965 & $1.29 \%$ & $1.36 \%$ \\
\hline 8 & 3070 & 1.0046 & $0.38 \%$ & $0.49 \%$ \\
\hline 9 & 1877 & 1.0058 & $0.73 \%$ & $2.06 \%$ \\
\hline 10 & 1296 & 1.0055 & $1.40 \%$ & $1.99 \%$ \\
\hline \hline
\end{tabular}

\section{B. Uncertainty Quantification}

Aerodynamic parameter uncertainties were estimated and characterized by using a "consider parameters" approach that is a variation of the minimum variance method. ${ }^{9}$ The noise-only covariance from Eq. (14), however, only relates the accuracy of the local parameter estimates with the accuracy of the observations. Put another way, it is a measure of how well the $M-\alpha-\beta$ solution fits the data, and does not take into account the accuracy of the global parameter estimates. By separately considering the effects of these additional estimates on the solution by considering them as random biases, the "consider parameters" approach provides more accurate uncertainties: 


$$
\Gamma_{\mathrm{x}, \text { consider }}=\Gamma_{\mathrm{x}}+\left(\mathrm{A}^{\mathrm{T}} \Gamma_{\varepsilon}^{-1} \mathrm{~A}\right)^{-1} \mathrm{~A}^{\mathrm{T}} \Gamma_{\varepsilon}^{-1} \mathrm{~B} \Gamma_{\mathrm{z}} \mathrm{B}^{\mathrm{T}} \Gamma_{\varepsilon}^{-1} \mathrm{~A}\left(\mathrm{~A}^{\mathrm{T}} \Gamma_{\varepsilon}^{-1} \mathrm{~A}\right)^{-1}
$$

where $\Gamma_{\mathrm{x}}$ is the covariance from Eq. (18), B is the sensitivity matrix of the global parameters (biases, scale factors, etc.), and $\Gamma_{\mathrm{z}}$ is the associated covariance. Figure 13 shows $1 \sigma$ uncertainties of the three estimated aerodynamic parameters. From the Figure, it can be seen that the uncertainties for the case with fewer ports used in the estimation (labeled "MEADS configuration") are higher than those for the case with three additional ports, as expected. Again recall that in both cases, data from ports 1 and 5 were excluded, and that any potential biases in the CFD database were absorbed by the estimated pressure transducer biases, scale factors, and higher order terms listed in Table 3 .
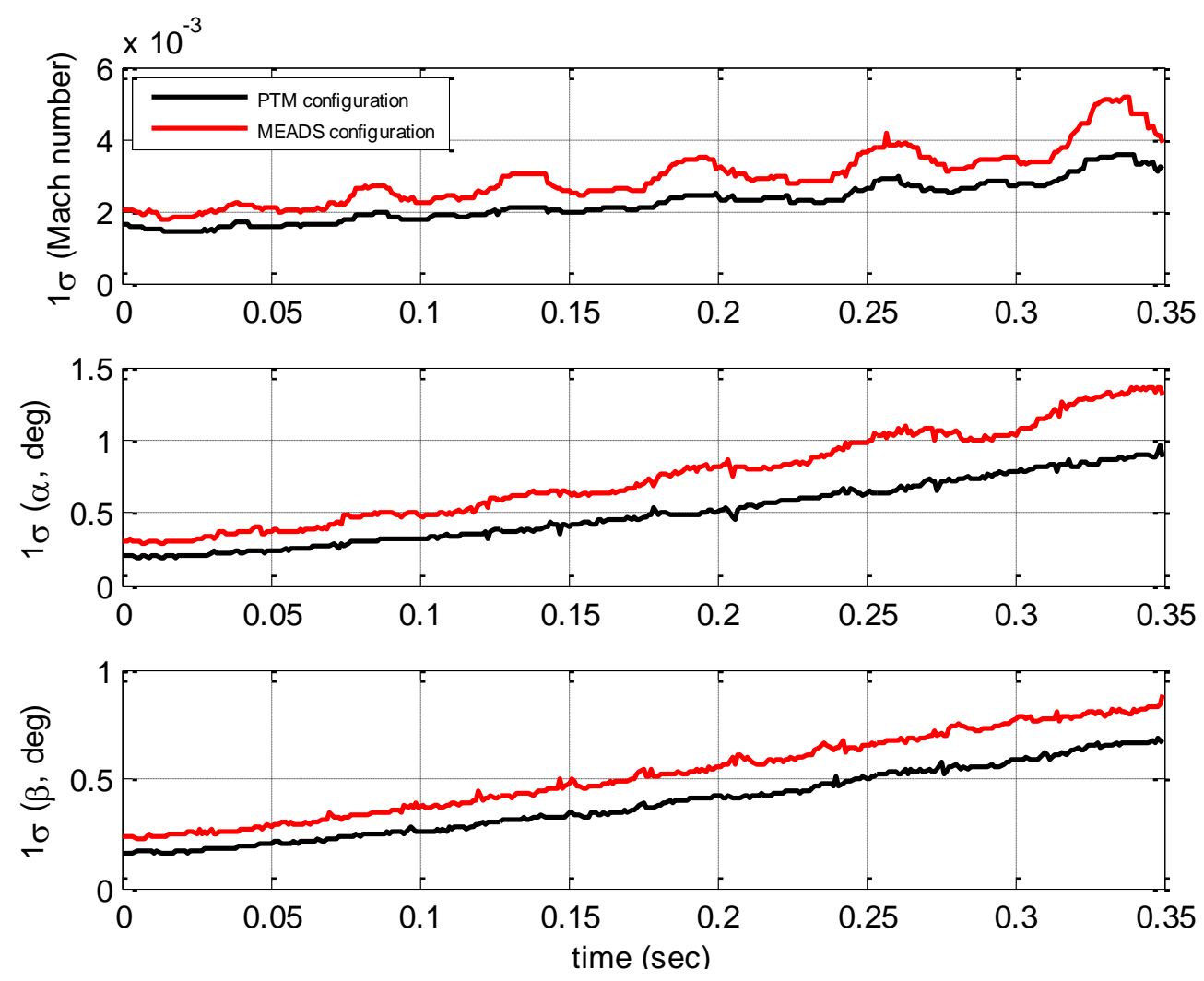

Figure 13. Aerodynamic parameter $1 \sigma$ uncertainties, MSL-PTM-01.

\section{Solution Validation}

Validation of the solution (inertial position, velocity, and aerodynamic parameters) was achieved by utilizing redundant data. For the MSL flight vehicle, this was done by comparing to landing site location from radiometry, altitude from radar, etc. For aeroballistic experiments, such validation was achieved in part from radar tracking data, as will be discussed in the present section.

\section{A. Mach Number Validation}

Mach number can be derived directly from the radar velocity from Eq. (6) and a vertical temperature profile from the meteorological data provided by weather balloons. It can also be derived using an iterative process from the Rayleigh-Pitot equation for the supersonic regime:

$$
R \equiv \frac{P_{\infty}}{P_{t}}=\left[\frac{2}{(\gamma+1) M_{\infty}^{2}}\right]^{\frac{\gamma}{\gamma-1}}\left[\frac{2 \gamma M_{\infty}^{2}-(\gamma-1)}{\gamma+1}\right]^{\frac{1}{\gamma-1}}
$$

The ratio $R$ computed from the RHS of this equation was compared to the value computed by the LHS ratio $P_{\infty} / P_{t}$, where the total pressure $P_{t}$ was assumed to be the maximum pressure observed by the pressure ports at any 
given time. The difference was then minimized in an iterative least squares sense to obtain the Mach number estimate at each observation time:

$$
\Delta M_{i}=\left[\frac{\partial R_{i}}{\partial M_{i}}\right]^{-1} R_{i}
$$

where $\partial R_{i} / \partial M_{i}$ is the sensitivity of $R$ with respect to $M$. Relative atmospheric humidity was taken into account when computing the specific heat ratio $\gamma$ using a polynomial model. ${ }^{13}$

Figure 14 shows the residuals between Mach number derived from radar velocity and Mach number from FADS and Rayleigh-Pitot. Biases are around 0.025, which are significantly larger than the $1 \sigma$ uncertainties from Figure 13. Potential error sources include measurement errors, CFD errors, errors in total pressure computation (recall that the total pressure is assumed to be the maximum pressure measured by any transducer at any given time, which may not be the true total pressure), and errors in the radar. Furthermore, a change in ambient pressure of 2000 $\mathrm{Pa}$ (less than the transducer uncertainty of $3000 \mathrm{~Pa}$ ), can produce a difference in the Rayleigh-Pitot Mach number estimate of up to 0.025 . In-barrel pressure readings just before firing varied between 1000 and $5000 \mathrm{~Pa}$ from ambient. The net change in $P_{\infty}$ across the first 0.35 seconds of flight, by contrast, was approximately $700 \mathrm{~Pa}$.

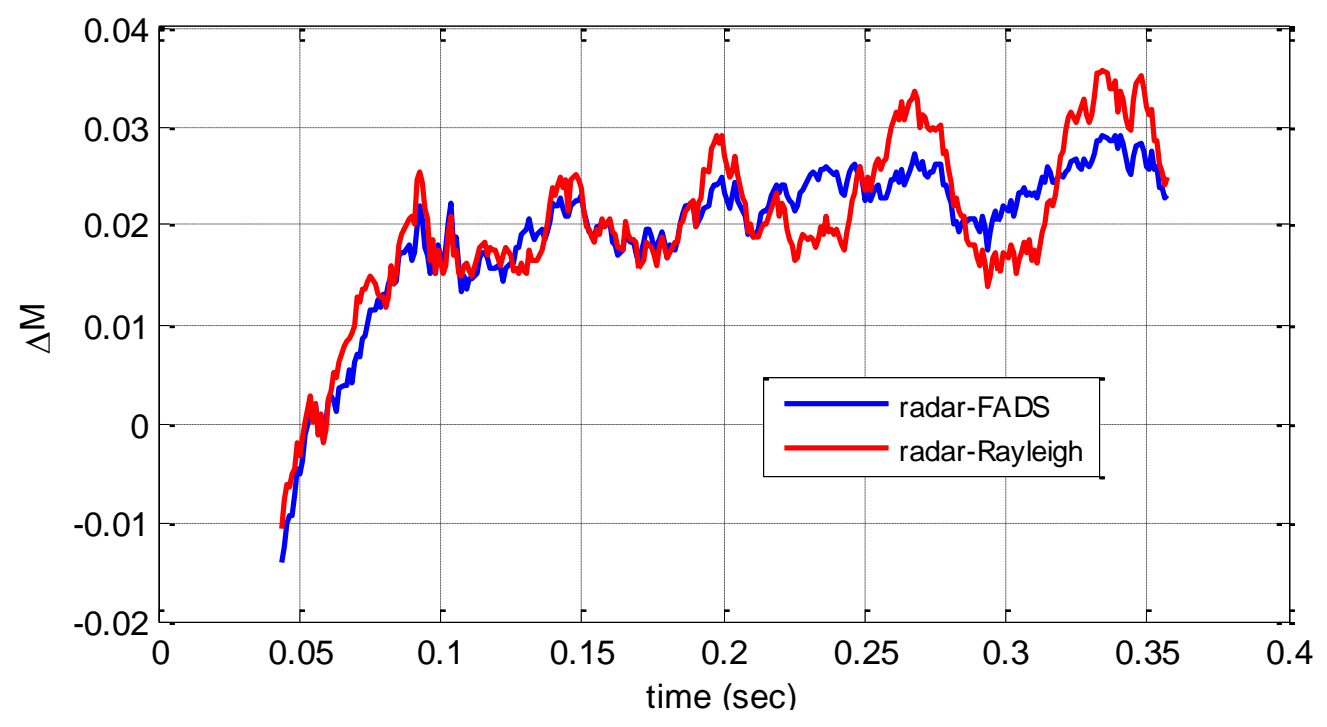

Figure 14. Mach number residuals, MSL-PTM-01

\section{B. Wind Angle Validation}

Since body frame velocities were not directly observable in these experiments, it was necessary to transform the radar inertial velocities to the body frame for an independent estimate of $\alpha$ and $\beta$ from Eq. (5). To do this, two vectors described in two frames were needed to create a direction cosine matrix (DCM), which can be computed using the triad method.

To compute a DCM from arbitrary vectors $\mathbf{x}$ and $\mathbf{y}$ in the body frame $b$ to the inertial frame $n$, it is necessary to form a third reference frame $z$, called the triad frame. To construct this frame, its first axis is defined as

$$
\begin{aligned}
\hat{\mathbf{z}}_{1}^{b} & =\hat{\mathbf{y}}^{b} \\
\hat{\mathbf{z}}_{1}^{n} & =\hat{\mathbf{y}}^{n}
\end{aligned}
$$

where $\hat{\mathbf{y}}^{b}$ and $\hat{\mathbf{y}}^{n}$ are the same unit vectors described in the body and inertial frames, respectively. The second axis is obtained by including the similarly-normalized vector $\mathbf{x}$ : 


$$
\begin{aligned}
\hat{\mathbf{z}}_{2}^{b} & =\frac{\hat{\mathbf{y}}^{b} \times \hat{\mathbf{x}}^{b}}{\left|\hat{\mathbf{y}}^{b} \times \hat{\mathbf{x}}^{b}\right|} \\
\hat{\mathbf{z}}_{2}^{n} & =\frac{\hat{\mathbf{y}}^{n} \times \hat{\mathbf{x}}^{n}}{\left|\hat{\mathbf{y}}^{n} \times \hat{\mathbf{x}}^{n}\right|}
\end{aligned}
$$

The final axis of the triad frame is the cross product of its first two axes:

$$
\begin{aligned}
\hat{\mathbf{z}}_{3}^{b} & =\hat{\mathbf{z}}_{1}^{b} \times \hat{\mathbf{z}}_{2}^{b} \\
\hat{\mathbf{z}}_{3}^{n} & =\hat{\mathbf{z}}_{1}^{n} \times \hat{\mathbf{z}}_{2}^{n}
\end{aligned}
$$

Rotation matrices are formed between the body frame $b$, inertial frame $n$, and the triad frame $z$ :

$$
\begin{aligned}
C^{z b} & =\left[\begin{array}{lll}
\hat{\mathbf{z}}_{1}^{b} & \hat{\mathbf{z}}_{2}^{b} & \hat{\mathbf{z}}_{3}^{b}
\end{array}\right] \\
C^{z n} & =\left[\begin{array}{lll}
\hat{\mathbf{z}}_{1}^{n} & \hat{\mathbf{z}}_{2}^{n} & \hat{\mathbf{z}}_{3}^{n}
\end{array}\right]
\end{aligned}
$$

where $C^{z b}$ is the DCM that transforms a vector from the body frame to the triad frame, and $C^{z n}$ is the DCM that transforms a vector from the inertial frame to the triad frame. The DCM that transforms a vector from the body frame to the inertial frame is therefore

$$
C^{n b}=\left[C^{z n}\right]^{\mathrm{T}} C^{z b}
$$

For the present analysis, the vectors used to compute these DCMs were acceleration measured by accelerometers and radar, and magnetic field direction and intensity measured by magnetometers. The magnetic field in the inertial frame was assumed to be constant, $\mathbf{B}^{n}=\left[\begin{array}{lll}-0.1837 & -0.4771 & 0.0969\end{array}\right]^{\mathrm{T}}$ gauss.

Once the history of DCMs that rotated a vector from the inertial frame to the body frame was obtained using these data, the (inertial) radar velocity vector was transformed to body frame $b$. From these radar-derived body velocities, the wind angles totally independent of the FADS solution were obtained from Eq. (5) and are shown in in Figure 15, with the FADS-based solutions shown for comparison. 

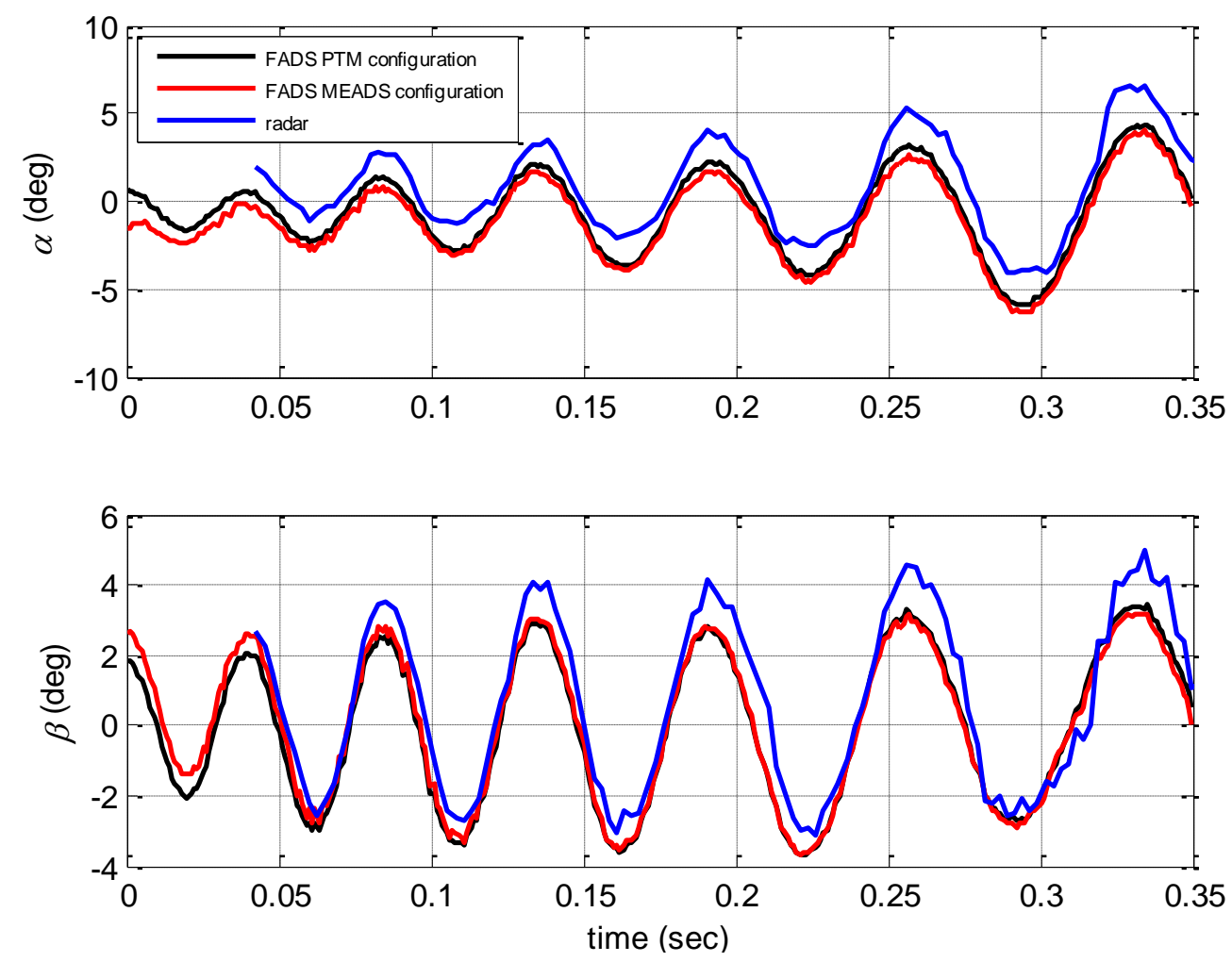

Figure 15. Wind angles, MSL-PTM-01. FADS solutions are repeated from Figure 11.

The expected trim angles for this non-lifting, axisymmetric flight were $0^{\circ}$ (the CFD database is symmetric at zero angle of attack and angle of sideslip). However, none of the estimated wind angles in Figure 15 trim precisely about zero. The biases in angle of attack may be attributed to accelerometer and/or magnetometer errors (since the radar-based solution uses these data), biases in the pressure data used to compute the FADS solution, and imperfections in the CG location of the PTM.

\section{Lifting Flights}

\section{A. Flight Overview and Discussion}

In addition to the non-lifting model MSL-PTM-01, two addition vehicles (MSL-PTM-02 and -03) were flown with offset CGs designed to mimic the MSL flight vehicle. Aside from these CG offsets, the vehicles carried identical instrumentation suites as the non-lifting model.

Figure 16 shows two sets of images taken from the high-speed tracking camera. The top set shows MSL-PTM02 as it exits the muzzle at right, then flies from right to left. Sabot fragments can be seen at the extreme right, and a bow shock can be seen in the second frame. The darkened area on the heat shield is the charred portion of the vehicle that was exposed to the in-barrel gases; the rest of the vehicle was encased in the sabot. A similar set of images is shown at the bottom of Figure 16 for MSL-PTM-03. 

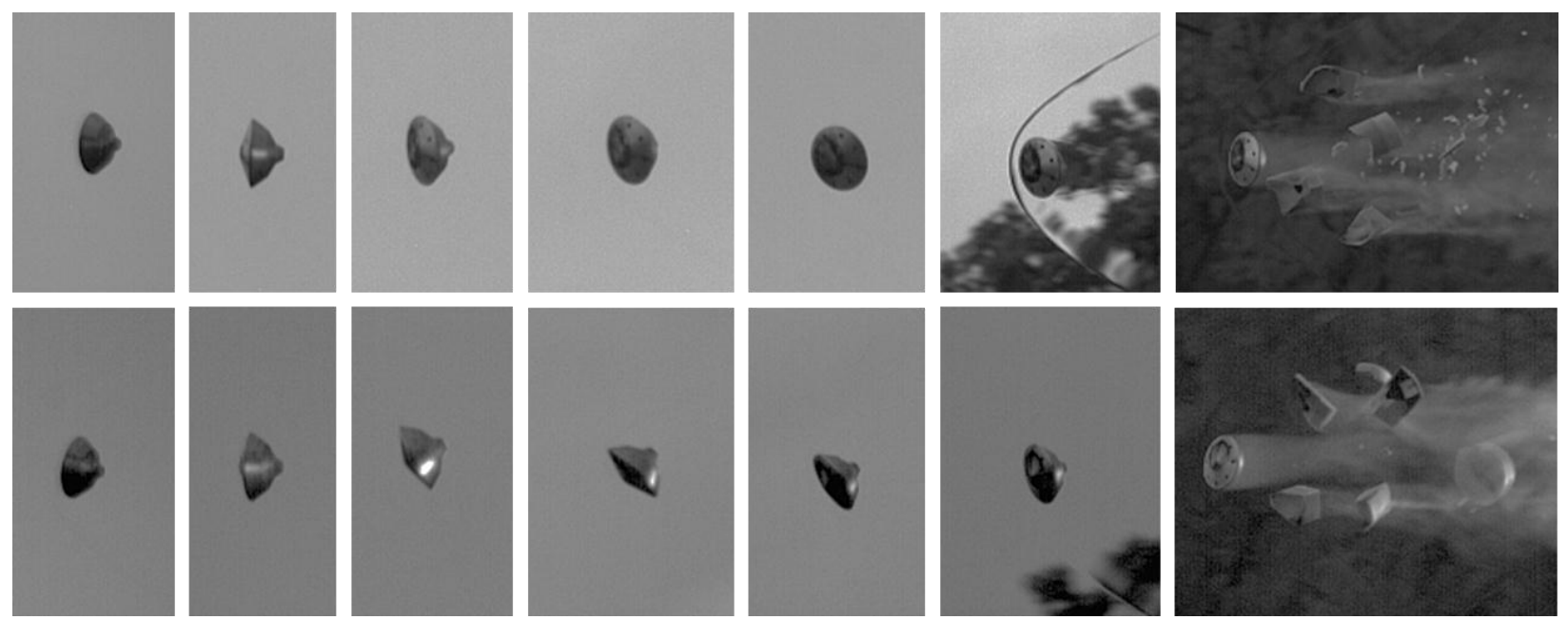

Figure 16. MSL-PTM-02 (top) and MSL-PTM-03 (bottom) imagery from high-speed flight follower camera. In both sets of images, the vehicle is moving from right to left, with muzzle exit at extreme right.

MSL-PTM-02 exited the muzzle at a high roll angle due to sabot/barrel interactions that caused a spin before muzzle exit. From high-speed camera imagery in Figure 16, it can be seen that the vehicle pitch plane (plane $x z$ in Figure 7) may be as much as $50^{\circ}$ off the vertical plane (plane $X Y$ in Figure 6). In addition, high-amplitude oscillations in the vehicle pitch plane are evident as the vehicle moves away from the gun; in fact, the vehicle heat shield faces the camera nearly head-on in the third frame from the right. At the time, it was believed that the sabot was "grabbing" the outer edge of the heat shield as it peeled away. This phenomenon was not observed in the smaller-scale $120 \mathrm{~mm}$ radar calibration firings, but may have been present to a lesser extent and was not detected because those models lacked any on-board instrumentation.

The sabot for the subsequent MSL-PTM-03 was modified to address this issue by removing sabot material on two petals to reduce contact area ahead of the model's maximum diameter. The limited number of sabots and models at the 7-inch scale, however, prevented testing or qualification of the modifications. Figure 17 shows this modification, as well as the portion of the heat shield that was exposed and later charred at the bottom right of Figure 16.

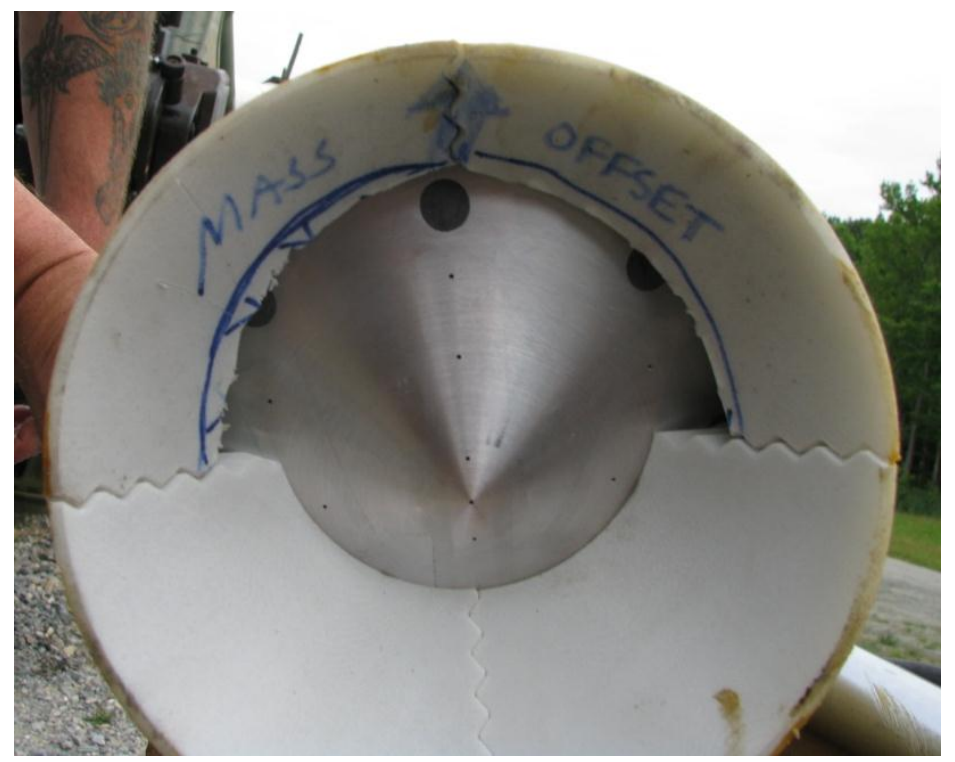

Figure 17. MSL-PTM lifting body encased in sabot. Sabot modification evident in reduced mass on upper half. Small black dots on vertical axis of heat shield (metallic portion) are FADS pressure ports. 
The extreme oscillation rates and accelerations on the lifting flights caused most of the IMU sensors to saturate over segments of oscillation cycles. These saturations prevented the computation of an inertial navigation solution. Figure 18 shows the body pitch rates measured by the IMU for MSL-PTM-02 and -03; MSL-PTM-01 is shown for comparison. The sensor saturation occurred at approximately 2100 degrees per second.

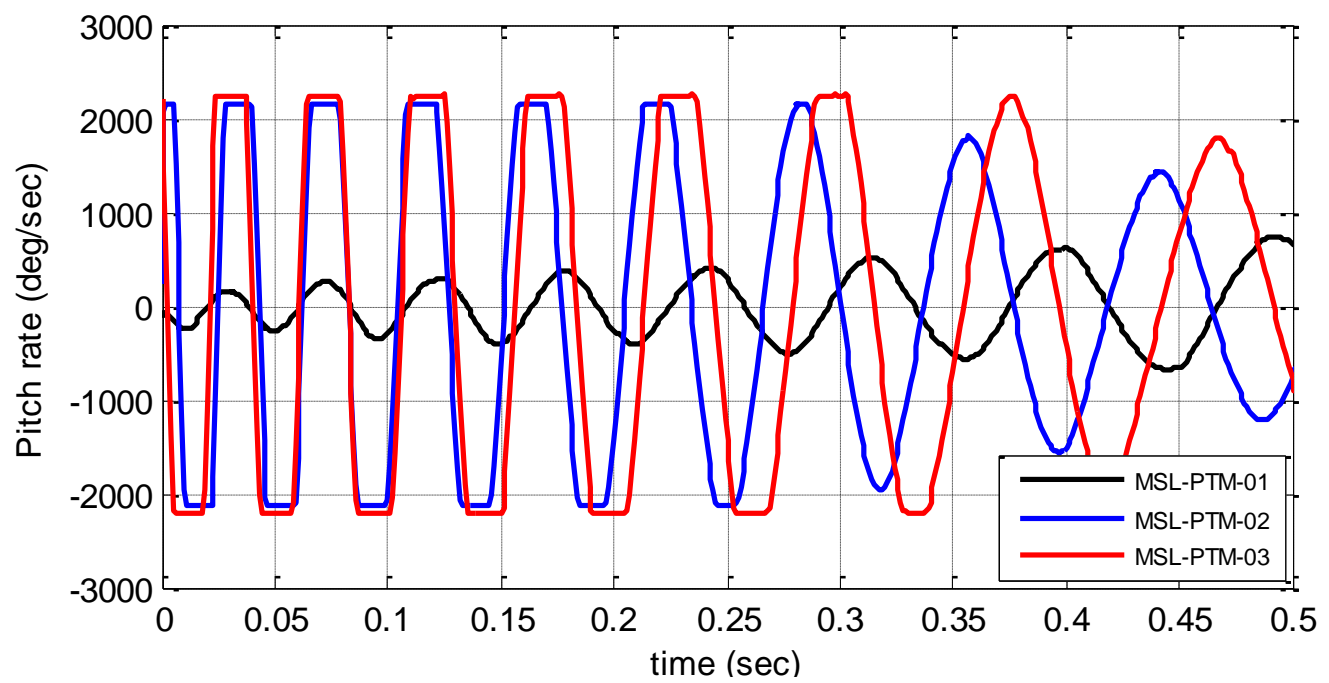

Figure 18. Body pitch rates measured by IMU.

A FADS-based solution using CFD was unable to be estimated because of the high pressure rates of change caused by these oscillations, and wind angles that exceeded the CFD database space. Figure 19 shows pressures measured by the transducers on MSL-PTM-02; compare to the pressures in Figure 10. Despite these issues, an attempt was made to use the validation techniques described in this paper to recover the aerodynamic parameters.
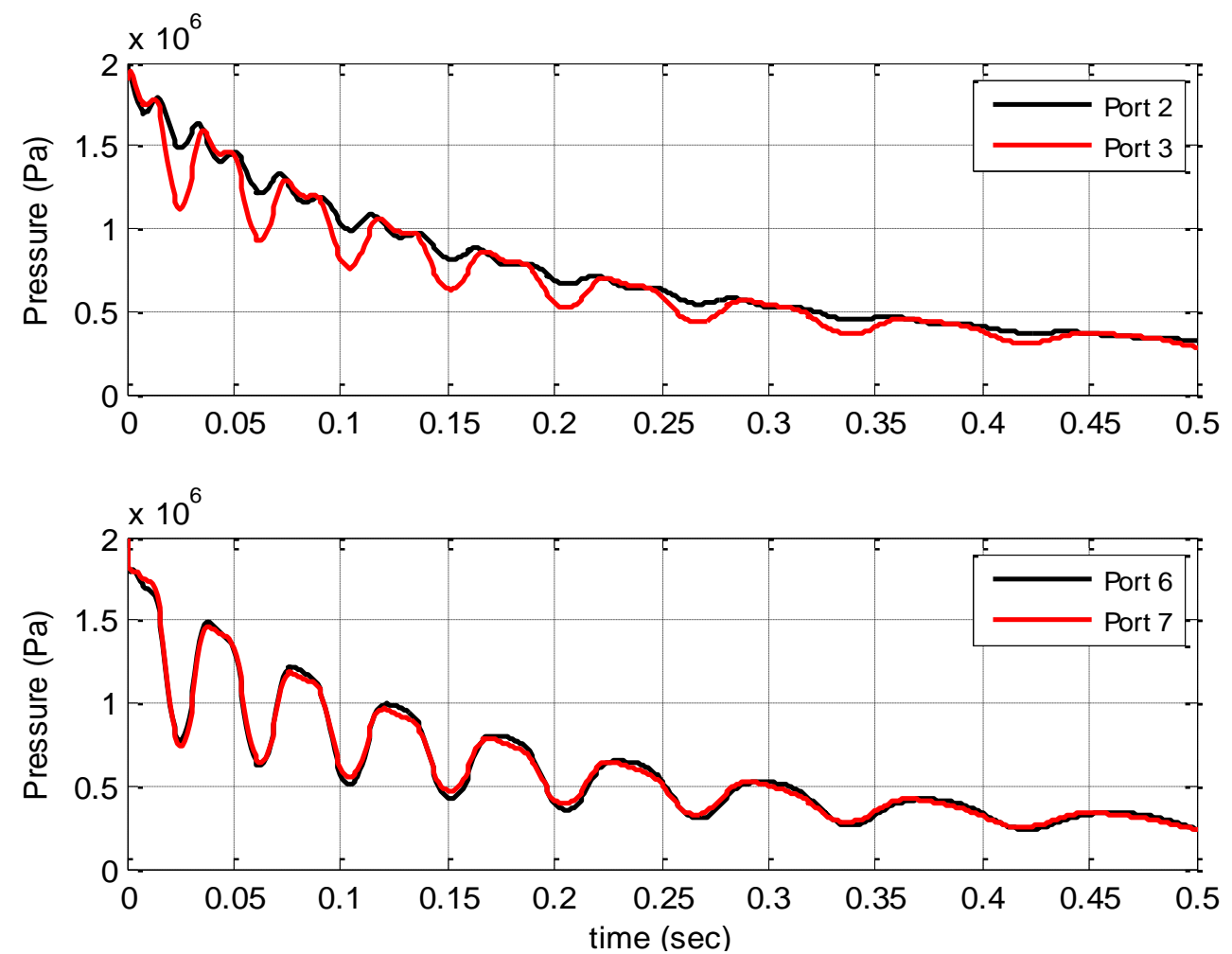

Figure 19. Observed FADS pressures, MSL-PTM-02.

17

American Institute of Aeronautics and Astronautics 


\section{B. Parameter Recovery}

Mach number was computed using the Rayleigh-Pitot technique in Eqs. (17-8) and is shown in Figure 20. Note that the segments of the Mach history that do not follow the smooth radar curve likely correspond to regions where the stagnation pressure was not located near any pressure transducers. Excluding these segments, which are shown by the blue lines at the bottom of Figure 20, the differences between radar and the Rayleigh-Pitot solutions are generally even smaller than those for MSL-PTM-01 in Figure 14.

Figure 21 shows the wind angles recovered from the triad method, using accelerations and magnetic field vectors in the body and inertial frames to obtain a direction cosine matrix history. The MSL-PTM-02 solution is of particular interest because it shows that the large initial oscillations continued to grow in amplitude as the vehicle slowed, until it reached an unstable point near $90^{\circ}$ at which point the vehicle flipped over.

The presence of an instability caused by high oscillation rates is reinforced by the high-speed flight follower video, which showed the vehicle pitching significantly immediately after muzzle exit, as well as exiting with a high roll angle (see Figure 16). This type of behavior was also seen in the CEV aeroballistic experiments, where the flight follower video showed a CEV PTM that had been launched with a non-zero sideslip angle very nearly tumble almost immediately after muzzle exit. ${ }^{9}$ MSL-PTM-03 also experienced high pitching rates similar to MSL-PTM-02 as shown in Figure 18, but did not tumble in first 1 second of flight, which was likely due to the fact that it was loaded in the gun with a non-zero roll angle to counteract the in-barrel rotation seen in MSL-PTM-02.
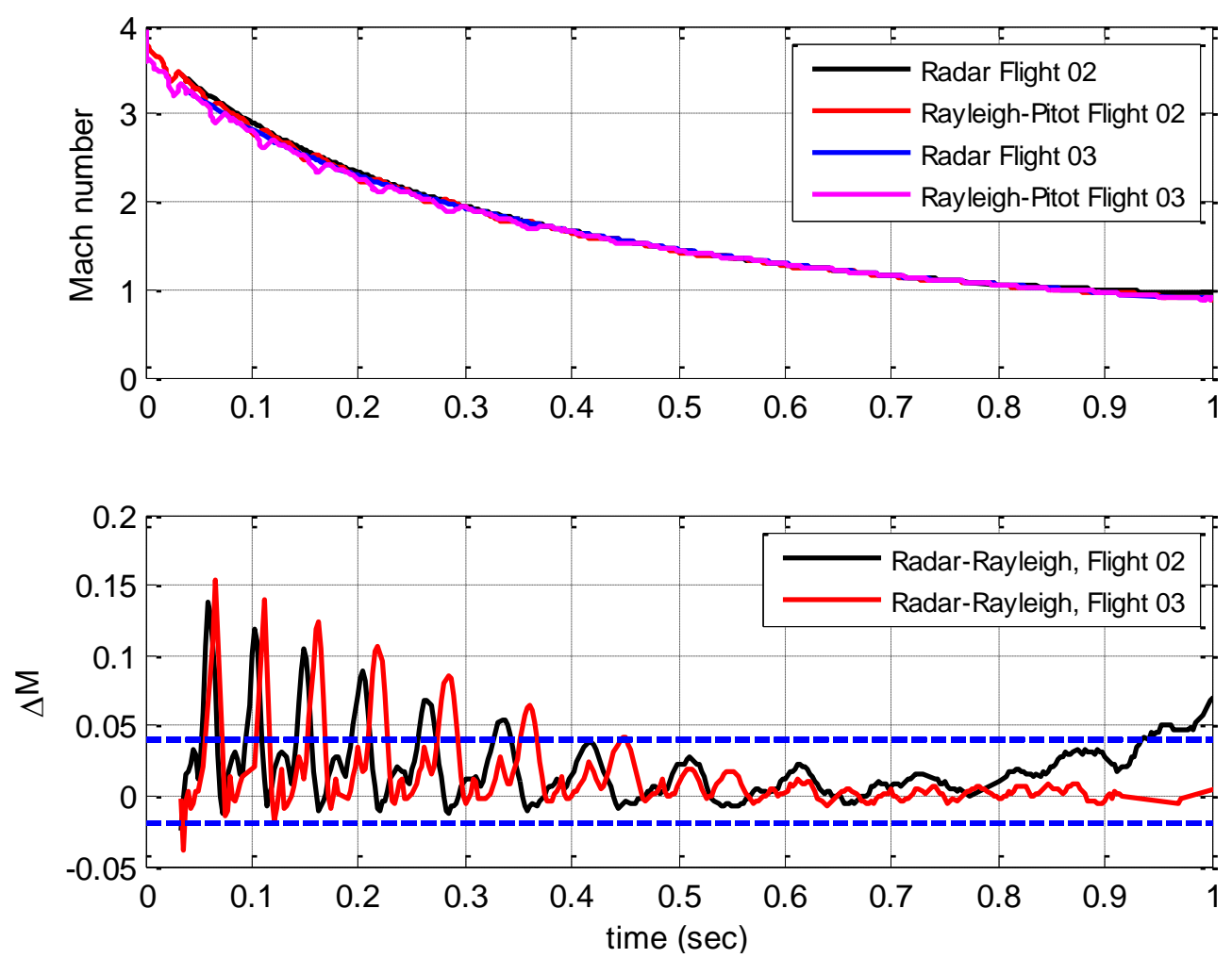

Figure 20. Mach number comparisons, lifting flights. 

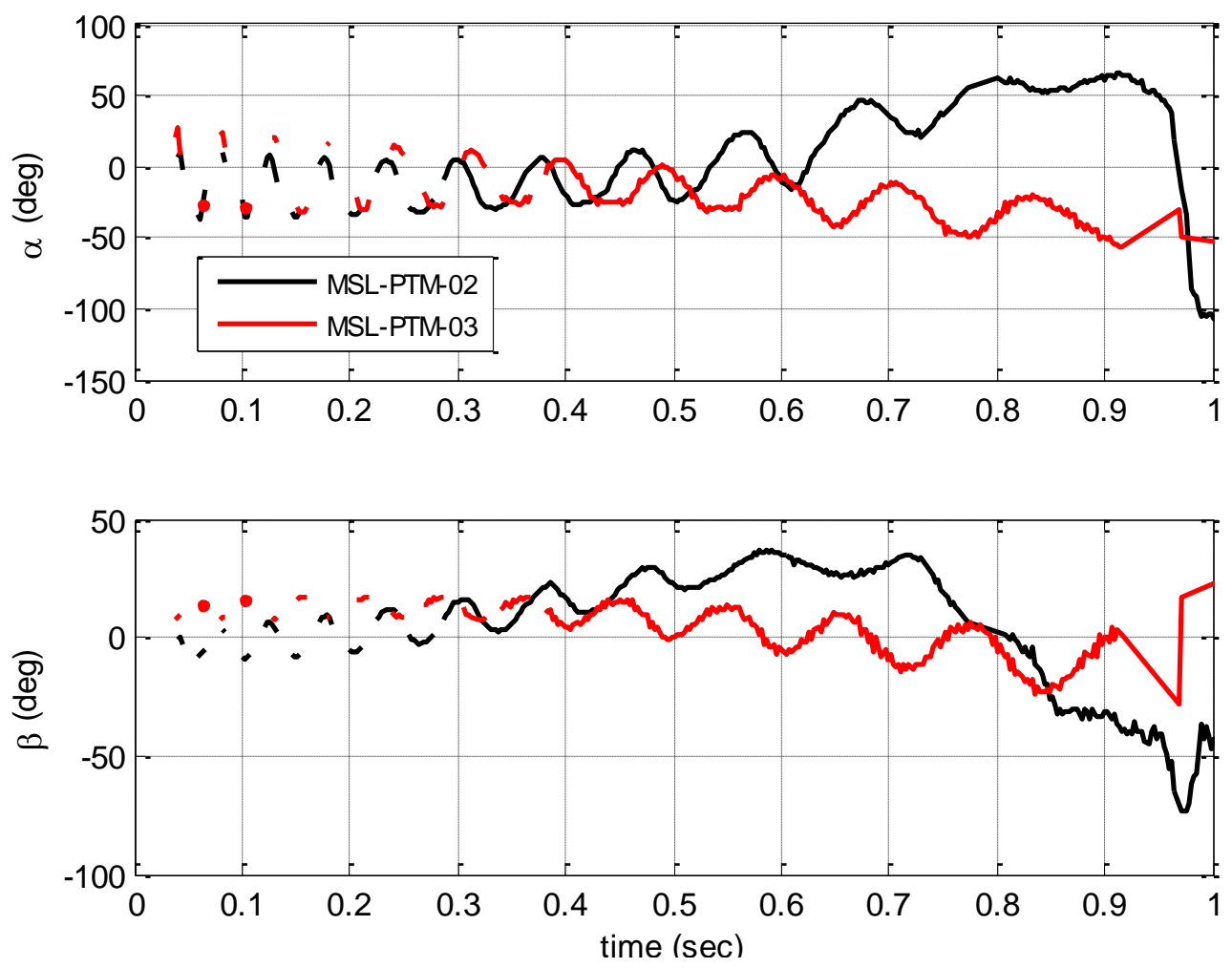

Figure 21. Wind angle history, MSL-PTM-02 and -03, from radar an, accelerometers, and magnetometers. Portions of flights that saturated IMU sensors are not shown.

\section{Summary and Conclusions}

Aeroballistic experiments were conducted using scale models of Mars Science Laboratory, with the goal of providing data types comparable to those of the MSL flight vehicle to the MSL trajectory reconstruction team. These models carried sensor compliments similar to those aboard the MSL flight vehicle, including an IMU and FADS. Redundant trajectory data were provided by on-board magnetometers and on-site tracking radar.

An inertial navigation trajectory was obtained using accelerometers and rate gyros, but was inaccurate due primarily to poorly-known initial conditions. The aerodynamic parameters Mach number, angle of attack, and sideslip angle were estimated using minimum variance with a priori to combine the pressure data and pre-flight CFD aerodynamics data. Both linear and non-linear pressure model terms were also estimated for each pressure transducer as a measure of the errors introduced by differences in transducer behavior between calibration and flight. Parameter uncertainties were estimated using a "consider parameters" approach, which provided a more realistic measure of the uncertainties than those associated with noise.

The FADS-based aerodynamic parameter estimates were validated independently of pressure and CFD data using IMU sensors and inertial position and velocity from radar data. This solution validation analysis showed that the differences between FADS- and radar-based aerodynamic parameter solutions exceed the $1 \sigma$ uncertainty estimates. Potential error sources include transducer biases, imperfections in the hardware, and modeling errors. The loss of two pressure ports along the vertical axis on MSL-PTM-01, which are primarily used to determine angle of attack, also contributed to differences that exceeded the $1 \sigma$ uncertainties by biasing the solutions.

For the lifting flights where FADS estimation methods were unable to be applied, validation techniques were still able to provide reasonable estimates of the aerodynamic parameters. Thus, even in a close to "worst-case" testing scenario where IMU and pressure data behavior render inertial navigation and FADS solutions impossible, redundant data sources from radar and magnetometers can still provide useful aerodynamic parameter estimates.

The vehicle/sabot interactions in the lifting flights that caused unexpectedly high oscillation rates did not appear in the $120 \mathrm{~mm}$ scale model radar calibration firings and may have been exacerbated by larger scales. The limited number of 7-inch models and accompanying sabots heavily restricted the qualification of mechanical solutions. In the case of MSL-PTM-02, the unstable flight was caused by high roll angle at muzzle exit coupled with abnormally 
high pitch rate amplitudes due to unexpected sabot/barrel and sabot/vehicle interactions, which indicate that sabot issues may cause experiment repeatability problems.

Future free-flight aeroballistic experiments would benefit from more extensive sabot qualification tests that simulate actual test conditions, e.g. similar scales and CG offsets. More economical alternatives may include using models instrumented only with accelerometers and magnetometers and tracking them with radar, and utilizing the validation techniques described in the present paper to recover aerodynamic parameters and qualify the sabot design and in-barrel behavior. Calibrating the pressure transducers over the entire pressure range that is expected to be observed would also aid in FADS solution accuracy, as would re-calibrating the transducers post-flight. The latter option would require the recovery of the model, which in the past has been prohibitive due to cost.

The results of the aeroballistic experiments show that useful pre-flight data can be acquired from aerodynamically scaled instrumented models in free flight. Combined with the "truth" trajectories provided by tracking radar and other redundant data sources, these data may be used to validate the tools and methodologies designed for trajectory reconstruction.

\section{Acknowledgements}

The authors wish to thank the following personnel for their time, effort, and support in preparing and conducting the aeroballistic experiments: Phil Peregino (ARL) for program management and coordination, Bernie Guidos (ARL) for experiment management and execution, Doug Petrick (ARL) for the PTM CAD/CAM mechanical designs, Brad Davis (ARL) for MIDAS sensor data acquisition and calibration, Phil Hufnal (Data Matrix) for mechanical drawing support, Kenny McMullen (ATC) for telemetry acquisition, Kenny Pugh (DSI/Bowhead) for MIDAS and pressure board assembly and calibration, Pete Jones (DSI/Bowhead) for radar calibration round fabrication, Walt Zdon (ATC) for radar data capture and reduction, Martin Chidsey (ATC) for flight follower video capture, Rick Moore, Lester Hitch, and Rashad Scott (Advanced Design Manufacturing) for rapid prototyping and fabrication of sabots, and the Transonic Experimental Facility gun crew.

\section{References}

${ }^{1}$ Gazarik, M. J., Wright, M. J., Little, A., Cheatwood, F. M., Herath, J. A., Munk, M. M., Novak, F. J., and Martinez, E. R., "Overview of the MEDLI Project," in IEEE 2008 Aerospace Conference, March 2008.

${ }^{2}$ Munk, M. M., Hutchinson, M., Mitchell, M., Parker, P., Little, A., Herath, J., Bruce, W., and Cheatwood, N., "Mars Entry Atmospheric Data System (MEADS): Requirements and Design for Mars Science Laboratory (MSL)," in 6th International Planetary Probe Workshop, Atlanta, GA, June 2008.

${ }^{3}$ Pruett, C. D., Wolf, H., Heck, M. L., and Siemers, P. M., “Innovative Air Data System for the Space Shuttle Orbiter," JSR, Vol. 20, No. 1, 1983, pp. 61-9.

${ }^{4}$ Siemers, P. M., Wolf, H., and Eades, J. B., "Shuttle Entry Air Data System (SEADS) - Advanced Air Data System Results: Air Data Across the Entry Speed Range,” NASA CP-3248, Part I, April 1995.

${ }^{5}$ Sebastian, T. and Tolson, R. H. "Methods for the Determination of Aerodynamic Parameters and Trajectory Reconstruction of the Orion Command Module from Scale Model Aeroballistic Flight Data," in 47th AIAA Aerospace Sciences Meeting Including The New Horizons Forum and Aerospace Exposition, Orlando, FL, 2009.

${ }^{6}$ Topper, B., Brown, T. G., Bukowski, E. F., Davis, B. S., Hall, R. A., Muller, P. C., Vong, T. T., and Brandon, F. J, "Feasibility of Determining Aerodynamic Coefficients for a NASA Apollo Body With the Use of Telemetry Data From Free Flight Range Testing," U.S. Army Research Laboratory, Aberdeen Proving Ground, MD, 2007, ARL-TR-4271.

${ }^{7}$ Murphy, C. M. and Bull, G. V., "Review of the High Altitude Research Program (HARP)," US Army Ballistic Research Lab, Aberdeen Proving Ground, MD, 1966, BRL-TR-1327.

${ }^{8}$ Muller, P., Davis, B., Condon, J., and Topper, B., "Characterizing Smart Munitions Flight Behavior with a Multifunctional Instrumentation and Data Acquisition System (MIDAS)," in ITEA Test Instrumentation Workshop, Las Vegas, NV, 2010.

${ }^{9}$ Lugo, R. A., "Methods of Flight Parameter Recovery from Orion Crew Module Using Scale Model Aeroballistic Flight Data," Department of Mechanical and Aerospace Engineering, North Carolina State University, Raleigh, NC, Master's Thesis 2011.

${ }^{10}$ Blanchard, R. C. and Desai, P. N., "Mars Phoenix Entry, Descent, and Landing Trajectory and Atmosphere Reconstruction," JSR, Vol. 48, No. 5, 2011, pp. 809-21.

${ }^{11}$ Karlgaard, C. D., "Mars Entry Atmospheric Data System Modeling and Algorithm Development," in 41st AIAA Thermophysics Conference, San Antonio, TX, 2009.

${ }^{12}$ Crassidis, J. L., and Junkins, J. L., Optimal Estimation of Dynamic Systems, Chapman \& Hall/CRC, Washington, D.C, 2004, Chaps. 1, 2.

${ }^{13}$ Cramer, O., "The variation of the specific heat ratio and the speed of sound in air with temperature, pressure, humidity, and $\mathrm{CO}_{2}$ concentration,” J. Acous. Soc. Am., Vol. 93, No. 5, 1993, pp. 2510-6. 